Article

\title{
Expanding the Mutation Spectrum in $A B C A 4:$ Sixty Novel Disease Causing Variants and Their Associated Phenotype in a Large French Stargardt Cohort
}

\author{
Marco Nassisi 1,2 (1), Saddek Mohand-Saï ${ }^{1,3}$, Claire-Marie Dhaenens ${ }^{4}$, Fiona Boyard ${ }^{1}$, \\ Vanessa Démontant ${ }^{1}$, Camille Andrieu ${ }^{3}$, Aline Antonio ${ }^{1}$, Christel Condroyer ${ }^{1}$, \\ Marine Foussard ${ }^{1}$, Cécile Méjécase ${ }^{1}$, Chiara Maria Eandi ${ }^{2}$, José-Alain Sahel ${ }^{1,3,5,6,7}$,

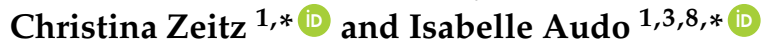 \\ 1 Sorbonne Université, INSERM, CNRS, Institut de la Vision, F-75012 Paris, France; \\ m.nassisi@gmail.com (M.N.); saddekms@gmail.com (S.M.-S.); fiona.boyard@gmail.com (F.B.); \\ vanessa.demontant@inserm.fr (V.D.); aline.antonio@inserm.fr (A.A.); christel.condroyer@inserm.fr (C.C.); \\ marine.foussard@inserm.fr (M.F.); cecile.mejecase@inserm.fr (C.M.); j.sahel@gmail.com (J.-A.S.) \\ Department of Surgical Sciences, Eye Clinic, University of Turin, 10126 Turin, Italy; chiara.eandi@unito.it \\ 3 Centre Hospitalier National d'Ophtalmologie des Quinze-Vingts, DHU Sight Restore, \\ INSERM-DHOS CIC 1423, F-75012 Paris, France; candrieu@15-20.fr \\ 4 Univ. Lille, Inserm UMR-S 1172, CHU Lille, Biochemistry and Molecular Biology Department-UF \\ Génopathies, F-59000 Lille, France; claire-marie.dhaenens@chru-lille.fr \\ 5 Fondation Ophtalmologique Adolphe de Rothschild, F-75019 Paris, France \\ 6 Académie des Sciences-Institut de France, F-75006 Paris, France \\ 7 Department of Ophthalmology, The University of Pittsburgh School of Medicine, Pittsburg, PA 15213, USA \\ 8 Institute of Ophthalmology, University College of London, London EC1V 9EL, UK \\ * Correspondence: christina.zeitz@inserm.fr (C.Z.); isabelle.audo@inserm.fr (I.A.)
}

Received: 21 May 2018; Accepted: 22 July 2018; Published: 27 July 2018

\begin{abstract}
Here we report novel mutations in $A B C A 4$ with the underlying phenotype in a large French cohort with autosomal recessive Stargardt disease. The DNA samples of 397 index subjects were analyzed in exons and flanking intronic regions of $A B C A 4$ (NM_000350.2) by microarray analysis and direct Sanger sequencing. At the end of the screening, at least two likely pathogenic mutations were found in 302 patients $(76.1 \%)$ while 95 remained unsolved: 40 (10.1\%) with no variants identified, $52(13.1 \%)$ with one heterozygous mutation, and $3(0.7 \%)$ with at least one variant of uncertain significance (VUS). Sixty-three novel variants were identified in the cohort. Three of them were variants of uncertain significance. The other 60 mutations were classified as likely pathogenic or pathogenic, and were identified in 61 patients (15.4\%). The majority of those were missense (55\%) followed by frameshift and nonsense (30\%), intronic (11.7\%) variants, and in-frame deletions $(3.3 \%)$. Only patients with variants never reported in literature were further analyzed herein. Recruited subjects underwent complete ophthalmic examination including best corrected visual acuity, kinetic and static perimetry, color vision test, full-field and multifocal electroretinography, color fundus photography, short-wavelength and near-infrared fundus autofluorescence imaging, and spectral domain optical coherence tomography. Clinical evaluation of each subject confirms the tendency that truncating mutations lead to a more severe phenotype with electroretinogram (ERG) impairment $(p=0.002)$ and an earlier age of onset $(p=0.037)$. Our study further expands the mutation spectrum in the exonic and flanking regions of $A B C A 4$ underlying Stargardt disease.
\end{abstract}

Keywords: $A B C A 4$; Stargardt disease; phenotype-genotype correlation 


\section{Introduction}

Stargardt disease-1 (STGD1, Mendelian Inheritance in Man [MIM] 248,200) is a progressive autosomal recessive macular degeneration linked to pathogenic mutations in $A B C A 4$ (MIM*601,691).

$A B C A 4$ encodes the transmembrane protein ABCA4 (ATP-binding cassette (ABC), subfamily A, member 4), located in the outer segment disk membranes of cone and rod photoreceptor cells [1-3]. Its loss can lead to the development of several retinal disorders including STGD1, cone and cone-rod dystrophy, and retinitis pigmentosa [4-11].

Given the large number of variants reported in $A B C A 4$ [12], most of them being polymorphisms, the identification of true disease-causing mutations is often challenging $[5,13,14]$.

With the advent of new analytical approaches, such as next generation sequencing (NGS), the detection rates of $A B C A 4$ mutations have greatly increased since its discovery in 1997. Nevertheless, homozygous or compound heterozygous mutations are regularly detected in no more than $70-75 \%$ of STGD1 patients, while a significant number of patients carry only a single $A B C A 4$ mutation or none at all [15-19]. On the other hand, several deep intronic variants in non-coding regions were reported to segregate with STGD1 affecting correct splicing mechanisms [19-23].

Interestingly, all genotyping studies on $A B C A 4$ report a broad mutation spectrum and a high allelic heterogeneity $[6,12,24-26]$. This phenomenon may be partially explained by the ethnic variability of the studied populations [12]. Therefore the need to screen large populations and to identify novel variants is still relevant $[27,28]$ and can help not only to explore the genetic architecture of $A B C A 4$ pathology, but also the genotype/phenotype correlation. Much effort today is directed towards a more specific correlation tailored on single variants [24,28-31], even though their low frequency makes it challenging. Reporting $A B C A 4$ novel variants with a clear and definite correlation with STGD1 would also help the proper selection of patients for therapeutic clinical trials reaching a higher degree of confidence with molecular diagnosis [32,33].

To further explore the genetic characteristics of $A B C A 4$ and broaden the spectrum of its pathogenic variants, we analyzed a large French cohort of 397 STGD1 patients using a combination of microarray analysis and Sanger sequencing. The purpose of this study was to report all the novel variants identified evaluating their pathogenicity with in silico analysis. The clinical features were also analyzed and correlated with the genetic results.

\section{Results}

Sequencing of $A B C A 4$ in our cohort of 397 STGD1 patients identified a large number of allelic variants. Microarray technology (ABCR600, ASPER Biotech, Inc., Tartu, Estonia) was used to screen 211 subjects for already known mutations, demonstrating at least two pathogenic variants in 76 of them. All the unsolved and the remaining cases were addressed to direct Sanger sequencing. At the end of the screening at least two likely pathogenic mutations were found in 302 patients $(76.1 \%)$, while 95 remained unsolved: $40(10.1 \%)$ with no variants identified, 52 (13.1\%) with one heterozygous mutation, and $3(0.7 \%)$ with at least one variant of uncertain significance (VUS) (Figure 1).

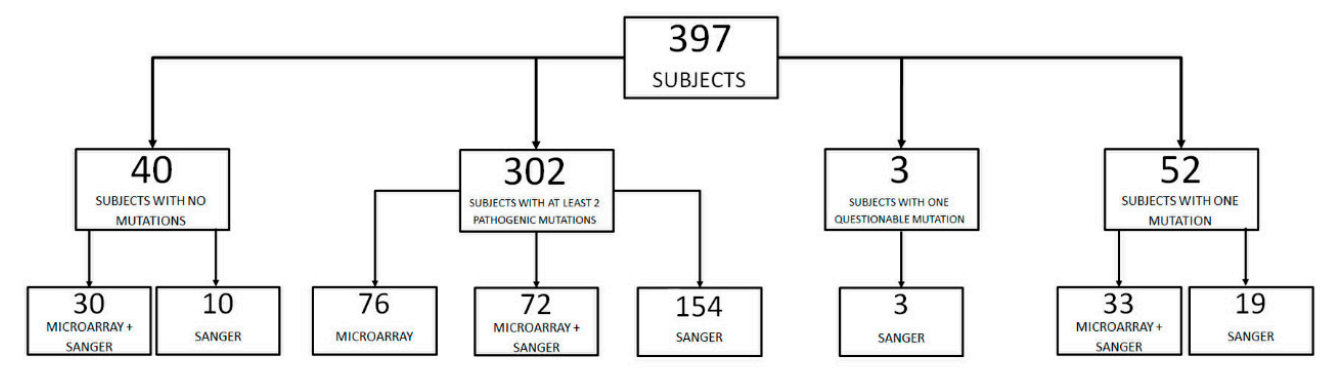

Figure 1. Genotypic analysis of a cohort of patients with clinical diagnosis of Stargardt disease using microarray analysis and/or direct Sanger sequencing of exonic and exon-flanking regions of $A B C A 4$. 


\subsection{Novel Variants}

Sixty-three novel $A B C A 4$ variants were identified in 63 index patients ( 30 males); 60 variants (in 61 subjects) were likely pathogenic or pathogenic. The other 3 variants (in 3 subjects) were VUS (Table 1, uncertain). CIC03678 was a carrier of two novel mutations, one likely pathogenic and one VUS.

The 60 likely pathogenic or pathogenic variants comprised 33 missense and 6 nonsense variants, 11 deletions, 1 one-base pair (bp) duplication, 1 four-bp duplication, 1 insertion, and 7 variants presumably affecting splicing (5 single nucleotide substitutions, 1 single-bp deletion, and 1 eight-bp deletion) (Table 1), which co-segregated with the phenotype in tested available family members.

Table 1. Sixty-three novel variants in patients with Stargardt disease and their classification. Nucleotide positions and translation correspond to CCDS747.1 and NP_000341.2, respectively.

\begin{tabular}{|c|c|c|c|}
\hline Exon/Intron & Variant & Protein Change & Classification \\
\hline 1 & c. $53 \mathrm{G}>\mathrm{A}$ & p.(Arg18Gln) & likely pathogenic \\
\hline IVS5 & c.570+1_570+8del & p.? & pathogenic \\
\hline 6 & c. $686 \mathrm{~T}>\mathrm{C}$ & p.(Leu229Pro) & likely pathogenic \\
\hline IVS7 & c. $859-2 A>G$ & p.? & pathogenic \\
\hline 8 & c.902del & p.(Arg301Serfs*15) & pathogenic \\
\hline 8 & c.972_973delinsAT & p. $\left(\right.$ Cys $\left.324^{*}\right)$ & pathogenic \\
\hline 8 & c. $978 \mathrm{C}>\mathrm{A}$ & p. $($ Tyr326*) & pathogenic \\
\hline 8 & c. $1019 A>G$ & p.(Tyr340Cys) & likely pathogenic \\
\hline 8 & c.1050del & p.(Ile351Leufs*23) & pathogenic \\
\hline IVS8 & c. $1099+1 G>C$ & p.? & pathogenic \\
\hline 9 & c. $1201 \mathrm{~A}>\mathrm{C}$ & p.(Thr401Pro) & likely pathogenic \\
\hline 10 & c. $1252 \mathrm{~T}>\mathrm{C}$ & p.(Phe418Leu) & likely pathogenic \\
\hline 10 & c. $1301 \mathrm{~T}>\mathrm{G}$ & p.(Val434Gly) & likely pathogenic \\
\hline 12 & c.1556G >A & p.(Cys519Tyr) & likely pathogenic \\
\hline 12 & c.1648_1659del & p.(Gly550_Phe553del) & likely pathogenic \\
\hline 12 & c. $1706 \mathrm{~A}>\mathrm{G}$ & p.(Tyr569Cys) & likely pathogenic \\
\hline 13 & c. $1895 \mathrm{~T}>\mathrm{A}$ & p.(Ile632Asn) & likely pathogenic \\
\hline 14 & c. $2083 \mathrm{G}>\mathrm{C}$ & p.(Val695Leu) & likely pathogenic \\
\hline 15 & c.2169_2172dup & p.(Leu725Asnfs $\left.{ }^{*} 42\right)$ & pathogenic \\
\hline 15 & c. $2299 \mathrm{del}$ & p.(Val767Serfs*20) & pathogenic \\
\hline 16 & c. $2443 \mathrm{C}>\mathrm{T}$ & p. $\left(\mathrm{G} \ln 815^{*}\right)$ & pathogenic \\
\hline 16 & c.2572dup & p.(Asp858Glyfs*27) & pathogenic \\
\hline 19 & c. $2868 \mathrm{C}>\mathrm{A}$ & p.(Asn956Lys) & uncertain \\
\hline 21 & c. $3080 \mathrm{~A}>\mathrm{G}$ & p.(Tyr1027Cys) & likely pathogenic \\
\hline 22 & c. $3311 \mathrm{~T}>\mathrm{C}$ & p.(Leu1104Pro) & likely pathogenic \\
\hline 23 & c. $3409 A>G$ & p.(Arg1137Gly) & uncertain \\
\hline 25 & c. $3682 \mathrm{G}>\mathrm{T}$ & p.(Glu1228*) & likely pathogenic \\
\hline 25 & c. $3811 G>C$ & p.(Glu1271Asp) & likely pathogenic \\
\hline 26 & c. $3825 G>C$ & p.(Lys1275Asn) & likely pathogenic \\
\hline 27 & c.3966del & p.(Ala1324Argfs*65) & pathogenic \\
\hline 27 & c. $4061 \mathrm{~A}>\mathrm{C}$ & p.(His1354Pro) & likely pathogenic \\
\hline IVS27 & c. $4129-3 \mathrm{C}>\mathrm{A}$ & p.? & likely pathogenic \\
\hline 28 & c.4178_4192del & p.(Val1393_Phe1397del) & likely pathogenic \\
\hline 29 & c. $4324 \mathrm{~A}>\mathrm{G}$ & p.(Asn1442Asp) & likely pathogenic \\
\hline 30 & c.4510_4535del & p.(Glu1504Profs*42) & pathogenic \\
\hline 32 & c.4663_4664del & p.(Gln1555Glufs*41) & pathogenic \\
\hline 33 & c. $4689 \mathrm{del}$ & p.(Gly1564Glufs*17) & pathogenic \\
\hline 33 & c. $4696 \mathrm{C}>\mathrm{T}$ & p.(Leu1566Phe) & likely pathogenic \\
\hline 33 & c. $4700 \mathrm{C}>\mathrm{T}$ & p.(Pro1567Leu) & likely pathogenic \\
\hline 34 & c. $4775 \mathrm{G}>\mathrm{A}$ & p.(Gly1592Asp) & likely pathogenic \\
\hline
\end{tabular}


Table 1. Cont.

\begin{tabular}{|c|c|c|c|}
\hline Exon/Intron & Variant & Protein Change & Classification \\
\hline 37 & c. $5282 C>G$ & p.(Pro1761Arg) & likely pathogenic \\
\hline 38 & c. $5332 \mathrm{~A}>\mathrm{T}$ & p.(Met1778Leu) & likely pathogenic \\
\hline 38 & c. $5342 \mathrm{C}>\mathrm{A}$ & p.(Ala1781Glu) & likely pathogenic \\
\hline 38 & c. $5351 \mathrm{~T}>\mathrm{C}$ & p.(Leu1784Pro) & likely pathogenic \\
\hline 38 & c. $5384 \mathrm{~T}>\mathrm{C}$ & p.(Leu1795Ser) & likely pathogenic \\
\hline 40 & c. $5621 \mathrm{~T}>\mathrm{C}$ & p.(Leu1874Pro) & likely pathogenic \\
\hline IVS42 & c. $5898+2 \mathrm{~T}>\mathrm{C}$ & p.? & pathogenic \\
\hline IVS42 & c. $5899-3 \mathrm{~T}>\mathrm{C}$ & p.? & uncertain \\
\hline 43 & c. 5939 C $>\mathrm{T}$ & p.(Thr1980Ile) & likely pathogenic \\
\hline IVS43 & c. $6005+1 \mathrm{del}$ & p.? & pathogenic \\
\hline 44 & c. $6110 \mathrm{C}>\mathrm{A}$ & p.(Ala2037Asp) & likely pathogenic \\
\hline 45 & c.6181_6184del & p.(Thr2061Serfs*53) & pathogenic \\
\hline 45 & c. $6191 \mathrm{C}>\mathrm{T}$ & p.(Ala2064Val) & likely pathogenic \\
\hline 45 & c. $6250 \mathrm{G}>\mathrm{A}$ & p.(Ala2084Thr) & likely pathogenic \\
\hline 46 & c. $6284 \mathrm{~A}>\mathrm{G}$ & p.(Asp2095Gly) & likely pathogenic \\
\hline 47 & c. $6394 \mathrm{G}>\mathrm{T}$ & p.(Glu2132*) & pathogenic \\
\hline 47 & c. $6454 \mathrm{G}>\mathrm{A}$ & p.(Gly2152Ser) & likely pathogenic \\
\hline 47 & c. $6455 \mathrm{G}>\mathrm{T}$ & p.(Gly2152Val) & likely pathogenic \\
\hline 47 & c.6436_6437insT & p.(Gly2146Valfs*36) & pathogenic \\
\hline 48 & c. $6693 \mathrm{del}$ & p.(Ile2231Metfs*16) & pathogenic \\
\hline 48 & c. $6704 \mathrm{C}>\mathrm{G}$ & p. $($ Ser2235*) & pathogenic \\
\hline 49 & c. $6746 \mathrm{C}>\mathrm{A}$ & p.(Ala2249Asp) & likely pathogenic \\
\hline IVS49 & c. $6816+1 G>T$ & p.? & pathogenic \\
\hline
\end{tabular}

Co-segregation analysis allowed the identification of complex alleles with in cis mutations in 18 patients. Nine additional patients had more than two variations, but it was impossible to establish phase and determine each allele due to the lack of DNA from additional family members.

All genotypic information about patients and co-segregation analysis are reported in Table 2.

Four missense mutations (i.e., p.(Cys519Tyr), p.(Glu1271Gln), p.(Gly1592Asp), and p.(Asp2095Gly)) were analyzed also with the splicing predictor tools due to their proximity with a putative splice site. None of them was predicted to affect splicing significantly but they were predicted as disease-causing by the missense prediction in silico algorithms.

Among all novel mutations, p.(Tyr340Cys), p.(Val434Gly), and p.(Gly2146Valfs*36) were found at a homozygous state (Table 2).

CIC08932 inherited the complex allele p.(Asn96Lys; Gly1961Glu) from the unaffected mother, but the novel variant p.(Gly1592Asp) was not found in the unaffected father (Table 2). This variant could be de novo in the index patient or CIC08933 may not be the biological father. Unfortunately, it was impossible to get this information from the subject, and our informed consent did not cover single nucleotide polymorphisms analysis for paternity testing.

Three novel variants found in three index patients (p.(Asn956Lys), p.(Arg1137Gly), and c.5899-3T>C) were considered VUS. Conservation and pathogenic prediction data for each variant are reported in Supplementary Tables S1 and S2. 
Table 2. Sixty novel likely pathogenic mutations in 61 index patients with Stargardt disease and cosegregation analysis. Nucleotide positions and translation correspond to CCDS747.1 and NP_000341.2, respectively.

\begin{tabular}{|c|c|c|c|c|c|c|c|c|c|c|}
\hline \multirow[b]{2}{*}{ Patient ID } & \multirow[b]{2}{*}{ Family ID } & & \multicolumn{3}{|c|}{ Allele 1} & \multicolumn{2}{|r|}{ Allele 2} & & \multicolumn{2}{|c|}{ Allele 1 or 2} \\
\hline & & & Exon/Intron & $\begin{array}{l}\text { Nucleotide } \\
\text { Change }\end{array}$ & Protein Change & Exon/Intron & $\begin{array}{l}\text { Nucleotide } \\
\text { Change }\end{array}$ & $\begin{array}{l}\text { Protein } \\
\text { Change }\end{array}$ & $\begin{array}{l}\text { Nucleotide } \\
\text { Change }\end{array}$ & $\begin{array}{l}\text { Protein } \\
\text { Change }\end{array}$ \\
\hline CIC03734 & 1673 & Index & 1 & c. $53 \mathrm{G}>\mathrm{A}$ & p.(Arg18Gln) & 42 & c.5882G>A [25] & p.(Gly1961Glu) & & \\
\hline CIC03735 & 1673 & $\begin{array}{l}\text { Affected } \\
\text { brother }\end{array}$ & 1 & c. $53 \mathrm{G}>\mathrm{A}$ & p.(Arg18Gln) & 42 & c. $5882 \mathrm{G}>\mathrm{A}[25]$ & p.(Gly1961Glu) & & \\
\hline CIC06131 & 1673 & $\begin{array}{l}\text { Unaffected } \\
\text { father }\end{array}$ & 1 & c. $53 \mathrm{G}>\mathrm{A}$ & p.(Arg18Gln) & & WILD TYPE & & & \\
\hline \multirow[t]{2}{*}{ CIC06908 } & 3783 & Index & IVS5 & c.570+1_570+8del & p.? & 40 & c.5603A $>$ T [34] & p.(Asn1868Ile) & & \\
\hline & & & 45 & c.6148G>C [25] & p.(Val2050Leu) & & & & & \\
\hline CIC08771 & 3783 & $\begin{array}{l}\text { Unaffected } \\
\text { mother }\end{array}$ & & WILD TYPE & & 40 & c. $5603 \mathrm{~A}>\mathrm{T}[34]$ & p.(Asn1868Ile) & & \\
\hline \multirow[t]{2}{*}{ CIC08774 } & 3783 & $\begin{array}{l}\text { Unaffected } \\
\text { brother }\end{array}$ & IVS5 & c. $570+1 \_570+8 \mathrm{del}$ & - & & WILD TYPE & & & \\
\hline & & & 45 & c.6148G>C [25] & p.(Val2050Leu) & & & & & \\
\hline \multirow[t]{2}{*}{ CIC07895 } & 4412 & Index & 6 & c. $686 \mathrm{~T}>\mathrm{C}$ & p.(Leu229Pro) & 24 & c.3602T>G [5] & p.(Leu1201Arg) & & \\
\hline & & & & & & 40 & c. $5621 \mathrm{~T}>\mathrm{C}$ & p.(Leu1874Pro) & & \\
\hline CIC07896 & 4412 & $\begin{array}{l}\text { Unaffected } \\
\text { mother }\end{array}$ & 6 & c. $686 \mathrm{~T}>\mathrm{C}$ & p.(Leu229Pro) & & WILD TYPE & & & \\
\hline \multirow[t]{2}{*}{ CIC07887 } & 4407 & Index & 6 & c. $686 \mathrm{~T}>\mathrm{C}$ & p.(Leu229Pro) & 42 & c.5882G>A [25] & p.(Gly1961Glu) & & \\
\hline & & & 48 & c.6529G >A [35] & p.(Asp2177Asn) & & & & & \\
\hline \multirow[t]{2}{*}{ CIC07888 } & 4407 & $\begin{array}{l}\text { Unaffected } \\
\text { mother }\end{array}$ & 6 & c. $686 \mathrm{~T}>\mathrm{C}$ & p.(Leu229Pro) & & WILD TYPE & & & \\
\hline & & & 48 & c.6529G >A [35] & p.(Asp2177Asn) & & & & & \\
\hline \multirow[t]{2}{*}{ CIC09848 } & 5658 & Index & IVS7 & c. $859-2 A>G$ & - & 8 & c. $872 \mathrm{C}>\mathrm{T}[36]$ & p.(Pro291Leu) & & \\
\hline & & & & & & 11 & c.1531C>T [18] & p.(Arg511Cys) & & \\
\hline \multirow[t]{2}{*}{ CIC09849 } & 5658 & $\begin{array}{l}\text { Unaffected } \\
\text { mother }\end{array}$ & & WILD TYPE & & 8 & c. $872 \mathrm{C}>\mathrm{T}[37]$ & p.(Pro291Leu) & & \\
\hline & & & & & & 11 & c.1531C>T [18] & p.(Arg511Cys) & & \\
\hline CIC09382 & 5385 & Index & 8 & c. $978 \mathrm{C}>\mathrm{A}$ & p.(Tyr326*) & IVS42 & c. $5898+2 \mathrm{~T}>\mathrm{C}$ & p.? & & \\
\hline CIC06749 & 3669 & Index & 8 & c.902del & p.(Arg301Serfs*15) & 46 & c.6320G>A [26] & p.(Arg2107His) & & \\
\hline
\end{tabular}


Table 2. Cont

\begin{tabular}{|c|c|c|c|c|c|c|c|c|c|c|c|}
\hline CIC06088 & 3203 & Index & 8 & c.972_973delinsAT & p. $($ Cys324 *) & 23 & c.3386G>T [38] & p.(Arg1129Leu) & & & \\
\hline CIC08477 & 3203 & $\begin{array}{l}\text { Unaffected } \\
\text { father }\end{array}$ & 8 & c.972_973delinsAT & p. $($ Cys324*) & & WILD TYPE & & & & \\
\hline CIC08478 & 3203 & $\begin{array}{l}\text { Unaffected } \\
\text { mother }\end{array}$ & & WILD TYPE & & 23 & c.3386G > T [38] & p.(Arg1129Leu) & & & \\
\hline CIC07725 & 4301 & Index & 8 & c.1019A >G & p.(Tyr340Cys) & 8 & c. $1019 A>G$ & p.(Tyr340Cys) & & & \\
\hline CIC07726 & 4301 & $\begin{array}{l}\text { Unaffected } \\
\text { father }\end{array}$ & 8 & c. $1019 A>G$ & p.(Tyr340Cys) & & WILD TYPE & & & & \\
\hline CICO7727 & 4301 & $\begin{array}{l}\text { Unaffected } \\
\text { mother }\end{array}$ & & WILD TYPE & & 8 & c. $1019 A>G$ & p.(Tyr340Cys) & & & \\
\hline CIC04235 & 2021 & Index & 8 & c.1050del & p.(Ile351Leufs*23) & 38 & c. $5351 \mathrm{~T}>\mathrm{C}$ & p.(Leu1784Pro) & & & \\
\hline CIC04236 & 2021 & $\begin{array}{c}\text { Unaffected } \\
\text { sister }\end{array}$ & 8 & c.1050del & p.(Ile351Leufs*23) & & WILD TYPE & & & & \\
\hline CIC07308 & 4026 & Index & 8 & c.1050del & p.(Ile351Leufs*23) & 42 & c.5882G>A [25] & p.(Gly1961Glu) & & & \\
\hline \multirow[t]{2}{*}{ CIC01080 } & 659 & Index & 8 & c.1050del & p.(Ile351Leufs*23) & 19 & c. $2819 C>G$ [39] & p.(Pro940Arg) & & & \\
\hline & & & & & & 23 & c.3364G>A [6] & p.(Glu1122Lys) & & & \\
\hline \multirow[t]{2}{*}{ CIC03521 } & 659 & $\begin{array}{l}\text { Unaffected } \\
\text { mother }\end{array}$ & & WILD TYPE & & 19 & c. $2819 C>G$ [39] & p.(Pro940Arg) & & & \\
\hline & & & & & & 23 & c.3364G>A [6] & p.(Glu1122Lys) & & & \\
\hline CIC03524 & 659 & $\begin{array}{l}\text { Unaffected } \\
\text { sister }\end{array}$ & & WILD TYPE & & & WILD TYPE & & & & \\
\hline CIC08372 & 4715 & Index & IVS8 & c.1099+1G>C & p.? & 22 & c.3322C>T [26] & p.(Arg1108Cys) & & & \\
\hline \multirow[t]{2}{*}{ CIC05266 } & 2672 & Index & 9 & c. $1201 \mathrm{~A}>\mathrm{C}$ & p.(Thr401Pro) & 17 & c. $2588 \mathrm{G}>\mathrm{C}[25]$ & p.(Gly863Ala) & & & \\
\hline & & & & & & 40 & c.5603A $>$ T [34] & p.(Asn1868Ile) & & & \\
\hline CIC05267 & 2672 & $\begin{array}{l}\text { Unaffected } \\
\text { mother }\end{array}$ & 9 & c. $1201 \mathrm{~A}>\mathrm{C}$ & p.(Thr401Pro) & & WILD TYPE & & & & \\
\hline \multirow[t]{2}{*}{ CIC05268 } & 2672 & $\begin{array}{l}\text { Unaffected } \\
\text { father }\end{array}$ & & WILD TYPE & & 17 & c. $2588 \mathrm{G}>\mathrm{C}[25]$ & p.(Gly863Ala) & & & \\
\hline & & & & & & 40 & c.5603A>T [34] & p.(Asn1868Ile) & & & \\
\hline CIC02804 & 1037 & Index & 10 & c. $1252 \mathrm{~T}>\mathrm{C}$ & p.(Phe418Leu) & IVS43 & c.6005+1del & p.? & 42 & c.5882G $>$ A [25] & p.(Gly1961Glu) \\
\hline CIC09625 & 5521 & Index & 10 & c. $1301 \mathrm{~T}>\mathrm{G}$ & p.(Val434Gly) & 10 & c. $1301 \mathrm{~T}>\mathrm{G}$ & p.(Val434Gly) & & & \\
\hline
\end{tabular}


Table 2. Cont

\begin{tabular}{|c|c|c|c|c|c|c|c|c|c|c|c|}
\hline CIC09624 & 5521 & $\begin{array}{c}\text { Affected } \\
\text { brother }\end{array}$ & 10 & c. $1301 \mathrm{~T}>\mathrm{G}$ & p.(Val434Gly) & 10 & c. $1301 \mathrm{~T}>\mathrm{G}$ & p.(Val434Gly) & & & \\
\hline CIC09626 & 5521 & $\begin{array}{l}\text { Unaffected } \\
\text { father }\end{array}$ & 10 & c. $1301 \mathrm{~T}>\mathrm{G}$ & p.(Val434Gly) & & WILD TYPE & & & & \\
\hline CIC09628 & 5521 & $\begin{array}{l}\text { Unaffected } \\
\text { mother }\end{array}$ & & WILD TYPE & & 10 & c. $1301 \mathrm{~T}>\mathrm{G}$ & p.(Val434Gly) & & & \\
\hline CIC01301 & 784 & Index & 12 & c.1556G >A & p.(Cys519Tyr) & 22 & c.3292C>T [40] & p.(Arg1098Cys) & & & \\
\hline CIC09999 & 784 & $\begin{array}{l}\text { Affected } \\
\text { brother }\end{array}$ & 12 & c. $1556 \mathrm{G}>\mathrm{A}$ & p.(Cys519Tyr) & 22 & c. $3292 \mathrm{C}>\mathrm{T}[41]$ & p.(Arg1098Cys) & & & \\
\hline CIC06346 & 3356 & Index & 12 & c.1648_1659del & p.(Gly550_Phe553del) & 42 & c. $5882 \mathrm{G}>\mathrm{A}[25]$ & p.(Gly1961Glu) & & & \\
\hline CIC08884 & 3356 & $\begin{array}{l}\text { Unaffected } \\
\text { father }\end{array}$ & 12 & c.1648_1659del & p.(Gly550_Phe553del) & & WILD TYPE & & & & \\
\hline CIC08949 & 3356 & $\begin{array}{l}\text { Unaffected } \\
\text { mother }\end{array}$ & & WILD TYPE & & 42 & c. $5882 \mathrm{G}>\mathrm{A}[25]$ & p.(Gly1961Glu) & & & \\
\hline CIC04197 & 1992 & Index & 12 & c. $1706 \mathrm{~A}>\mathrm{G}$ & p.(Tyr569Cys) & 40 & c.5603A>T [34] & p.(Asn1868Ile) & & & \\
\hline CIC07749 & 4314 & Index & 13 & c. $1895 \mathrm{~T}>\mathrm{A}$ & p.(Ile632Asn) & 33 & c. $4700 \mathrm{C}>\mathrm{T}$ & p.(Pro1567Leu) & 24 & c.3602T>G [5] & p.(Leu1201Arg) \\
\hline \multirow[t]{2}{*}{ CIC08724 } & 4948 & Index & 14 & c. $2083 G>C$ & p.(Val695Leu) & 47 & c. $6445 \mathrm{C}>\mathrm{T}[6]$ & p.(Arg2149 & & & \\
\hline & & & 44 & c.6079C $>$ T [29] & p.(Leu2027Phe) & & & & & & \\
\hline \multirow[t]{2}{*}{ CIC08723 } & 4948 & $\begin{array}{l}\text { Affected } \\
\text { sister }\end{array}$ & 14 & c. $2083 \mathrm{G}>\mathrm{C}$ & p.(Val695Leu) & 47 & c. $6445 \mathrm{C}>\mathrm{T}[6]$ & p.(Arg2149 & & & \\
\hline & & & 44 & c.6079C >T [29] & p.(Leu2027Phe) & & & & & & \\
\hline \multirow[t]{2}{*}{ CIC08858 } & 4948 & $\begin{array}{l}\text { Unaffected } \\
\text { mother }\end{array}$ & 14 & c. $2083 \mathrm{G}>\mathrm{C}$ & p.(Val695Leu) & & WILD TYPE & & & & \\
\hline & & & 44 & c.6079C $>$ T [29] & p.(Leu2027Phe) & & & & & & \\
\hline CIC08859 & 4948 & $\begin{array}{l}\text { Unaffected } \\
\text { father }\end{array}$ & & WILD TYPE & & 47 & c. $6445 \mathrm{C}>\mathrm{T}[6]$ & $\begin{array}{c}\text { p.(Arg2149 } \\
*)\end{array}$ & & & \\
\hline \multirow[t]{2}{*}{ CIC07985 } & 4793 & Index & 15 & c.2169_2172dup & p.(Leu725Asnfs*42) & 47 & c. $6454 \mathrm{G}>\mathrm{A}$ & p.(Gly2152Ser) & 44 & c. $6079 \mathrm{C}>\mathrm{T}[25]$ & p.(Leu2027Phe) \\
\hline & & & & & & & & & 40 & c.5603A>T [34] & p.(Asn1868Ile) \\
\hline CIC06126 & 3227 & Index & 15 & c.2299del & p.(Val767Serfs*20) & 17 & c. $2588 \mathrm{G}>\mathrm{C}[25]$ & p.(Gly863Ala) & 40 & c. $5603 \mathrm{~A}>\mathrm{T}[34]$ & p.(Asn1868Ile) \\
\hline CIC09405 & 5401 & Index & 16 & c. $2443 \mathrm{C}>\mathrm{T}$ & p. $\left(\mathrm{G} \ln 815^{*}\right)$ & 42 & c.5882G>A [25] & p.(Gly1961Glu) & & & \\
\hline
\end{tabular}


Table 2. Cont

\begin{tabular}{|c|c|c|c|c|c|c|c|c|c|c|}
\hline CIC09407 & 5401 & $\begin{array}{l}\text { Unaffected } \\
\text { mother }\end{array}$ & & WILD TYPE & & 42 & c. $5882 \mathrm{G}>\mathrm{A}[25]$ & p.(Gly1961Glu) & & \\
\hline CIC09408 & 5401 & $\begin{array}{l}\text { Unaffected } \\
\text { father }\end{array}$ & 16 & c. $2443 \mathrm{C}>\mathrm{T}$ & p. $\left(\mathrm{G} \ln 815^{*}\right)$ & & WILD TYPE & & & \\
\hline \multirow[t]{2}{*}{ CIC00467 } & 319 & Index & 16 & c.2572dup & p.(Asp858Glyfs*27) & 35 & c. $4926 \mathrm{C}>\mathrm{G}[42]$ & p.(Ser1642Arg) & & \\
\hline & & & & & & 36 & $\begin{array}{c}\text { c.5044_5058del } \\
{[25]}\end{array}$ & p.(Val1682_Val1686del) & & \\
\hline CIC05014 & 319 & $\begin{array}{l}\text { Unaffected } \\
\text { sister }\end{array}$ & 16 & c.2572dup & p.(Asp858Glyfs*27) & & WILD TYPE & & & \\
\hline \multirow[t]{2}{*}{ CIC05056 } & 319 & $\begin{array}{l}\text { Affected } \\
\text { brother }\end{array}$ & 16 & c.2572dup & p.(Asp858Glyfs*27) & 35 & c.4926C>G [43] & p.(Ser1642Arg) & & \\
\hline & & & & & & 36 & $\begin{array}{c}\text { c.5044_5058del } \\
\text { [25] }\end{array}$ & p.(Val1682_Val1686del) & & \\
\hline${ }^{+} \mathrm{CIC} 03678$ & 1627 & Index & 19 & c. $2868 \mathrm{C}>\mathrm{A}$ & p.(Asn956Lys) & 43 & c. $5939 \mathrm{C}>\mathrm{T}$ & p.(Thr1980Ile) & & \\
\hline CIC03679 & 1627 & $\begin{array}{l}\text { Unaffected } \\
\text { son }\end{array}$ & & WILD TYPE & & 43 & c. $5939 \mathrm{C}>\mathrm{T}$ & p.(Thr1980Ile) & & \\
\hline CIC04259 & 2036 & Index & 21 & c. $3080 A>G$ & p.(Tyr1027Cys) & 38 & c.5381C $>\mathrm{A}[24]$ & p.(Ala1794Asp) & & \\
\hline CIC08538 & 4826 & Index & 22 & c. $3311 \mathrm{~T}>\mathrm{C}$ & p.(Leu1104Pro) & IVS49 & $\begin{array}{c}\text { c.6816+1G }>A \\
{[6]}\end{array}$ & - & & \\
\hline CIC04176 & 1973 & Index & 25 & c. $3682 G>T$ & p.(Glu1228*) & 22 & c. $3322 \mathrm{C}>\mathrm{T}[26]$ & p.(Arg1108Cys) & & \\
\hline CIC02505 & 871 & Index & 25 & c. $3811 G>C$ & p.(Glu1271Gln) & 23 & c.3386G $>$ T [38] & p.(Arg1129Leu) & & \\
\hline CIC05899 & 3087 & Index & 25 & c. $3811 G>C$ & p.(Glu1271Gln) & 42 & c.5882G>A [25] & p.(Gly1961Glu) & & \\
\hline \multirow[t]{2}{*}{ CIC07120 } & 3909 & Index & 26 & c. $3825 \mathrm{G}>\mathrm{C}$ & p.(Lys1275Asn) & 22 & c. $3322 \mathrm{C}>\mathrm{T}[26]$ & p.(Arg1108Cys) 40 & c.5603A>T [34] & p.(Asn1868Ile) \\
\hline & & & & & & & & 46 & c.6320G >A [26] & p.(Arg2107His) \\
\hline \multirow[t]{2}{*}{ CIC01750 } & 1242 & Index & 27 & c.3966del & p.(Ala1324Argfs*65) & 35 & c.4918C $>$ T [26] & p.(Arg1640Trp) & & \\
\hline & & & & & & 40 & c. $5603 \mathrm{~A}>\mathrm{T}[34]$ & p.(Asn1868Ile) & & \\
\hline \multirow[t]{2}{*}{ CIC02024 } & 1242 & $\begin{array}{l}\text { Affected } \\
\text { sister }\end{array}$ & 27 & c.3966del & p.(Ala1324Argfs*65) & 35 & c.4918C > T [26] & p.(Arg1640Trp) & & \\
\hline & & & & & & 40 & c.5603A $>$ T [34] & p.(Asn1868Ile) & & \\
\hline \multirow[t]{2}{*}{ CIC07100 } & 1242 & $\begin{array}{l}\text { Unaffected } \\
\text { father }\end{array}$ & & WILD TYPE & & 35 & c. $4918 \mathrm{C}>\mathrm{T}[26]$ & p.(Arg1640Trp) & & \\
\hline & & & & & & 40 & c.5603A > T [34] & p.(Asn1868Ile) & & \\
\hline
\end{tabular}


Table 2. Cont

\begin{tabular}{|c|c|c|c|c|c|c|c|c|c|c|c|}
\hline CIC07146 & 1242 & $\begin{array}{l}\text { Unaffected } \\
\text { mother }\end{array}$ & 27 & c.3966del & p.(Ala1324Argfs*65) & & WILD TYPE & & & & \\
\hline \multirow[t]{2}{*}{ CIC07744 } & 4372 & Index & 27 & c. $4061 \mathrm{~A}>\mathrm{C}$ & p.(His1354Pro) & 13 & c.1927G>A [44] & p.(Val643Met) & 8 & c.872C >T [37] & p.(Pro291Leu) \\
\hline & & & & & & & & & 24 & c.3602T>G [6] & p.(Leu1201Arg) \\
\hline \multirow[t]{2}{*}{ CIC02690 } & 961 & Index & IVS27 & c. $4129-3 \mathrm{C}>\mathrm{A}$ & p.? & IVS38 & $\begin{array}{c}\text { c. } 5461-10 \mathrm{~T}>\mathrm{C} \\
{[45]}\end{array}$ & $\begin{array}{c}\text { p. } \\
\text { [Thr1821Valfs, } \\
\text { Thr1821Aspfs] }\end{array}$ & & & \\
\hline & & & & & & 40 & c.5603A>T [34] & p.(Asn1868Ile) & & & \\
\hline \multirow[t]{2}{*}{ CIC02691 } & 961 & $\begin{array}{l}\text { Unaffected } \\
\text { mother }\end{array}$ & & WILD TYPE & & IVS38 & $\begin{array}{c}\text { c. } 5461-10 \mathrm{~T}>\mathrm{C} \\
{[45]}\end{array}$ & $\begin{array}{c}\text { p. } \\
\text { [Thr1821Valfs, } \\
\text { Thr1821Aspfs] }\end{array}$ & & & \\
\hline & & & & & & 40 & c.5603A>T [34] & p.(Asn1868Ile) & & & \\
\hline CIC08194 & 4588 & Index & 28 & c. $4178 \_4192 \mathrm{del}$ & p.(Val1393_Phe1397del) & 40 & c. $5603 \mathrm{~A}>\mathrm{T}[34]$ & p.(Asn1868Ile) & 45 & c. $6148 \mathrm{G}>\mathrm{C}[25]$ & p.(Val2050Leu) \\
\hline CIC07994 & 4463 & Index & 29 & c. $4324 \mathrm{~A}>\mathrm{G}$ & p.(Asn1442Asp) & 3 & c.194G>A [46] & p.(Gly65Glu) & & & \\
\hline CIC07998 & 4463 & $\begin{array}{l}\text { Unaffected } \\
\text { mother }\end{array}$ & & WILD TYPE & & 3 & c.194G>A [46] & p.(Gly65Glu) & & & \\
\hline CIC06727 & 3652 & Index & 30 & c.4510_4535del & p.(Glu1504Profs*42) & 28 & c.4139C>T [6] & p.(Pro1380Leu) & & & \\
\hline CIC06728 & 3652 & $\begin{array}{l}\text { Unaffected } \\
\text { father }\end{array}$ & & WILD TYPE & & 28 & c. $4139 \mathrm{C}>\mathrm{T}[6]$ & p.(Pro1380Leu) & & & \\
\hline CIC06729 & 3652 & $\begin{array}{c}\text { Unaffected } \\
\text { brother }\end{array}$ & & WILD TYPE & & 28 & c. $4139 \mathrm{C}>\mathrm{T}[6]$ & p.(Pro1380Leu) & & & \\
\hline CIC06730 & 3652 & $\begin{array}{l}\text { Unaffected } \\
\text { mother }\end{array}$ & 30 & c.4510_4535del & p.(Glu1504Profs*42) & & WILD TYPE & & & & \\
\hline CIC08283 & 4647 & Index & 32 & c.4663_4664del & p.(Gln1555Glufs*41) & 40 & c.5603A>T [34] & p.(Asn1868Ile) & & & \\
\hline \multirow[t]{2}{*}{ CIC03252 } & 1375 & Index & 33 & c.4689del & p.(Gly1564Glufs*17) & 17 & c. $2588 \mathrm{G}>\mathrm{C}[25]$ & p.(Gly863Ala) & & & \\
\hline & & & & & & 40 & c.5603A $>\mathrm{T}[34]$ & p.(Asn1868Ile) & & & \\
\hline \multirow[t]{2}{*}{ CIC03253 } & 1375 & $\begin{array}{l}\text { Unaffected } \\
\text { mother }\end{array}$ & & WILD TYPE & & 17 & c. $2588 \mathrm{G}>\mathrm{C}[25]$ & p.(Gly863Ala) & & & \\
\hline & & & & & & 40 & c.5603A $>\mathrm{T}[34]$ & p.(Asn1868Ile) & & & \\
\hline \multirow[t]{2}{*}{ CIC09219 } & 5282 & Index & 33 & c. $4696 \mathrm{C}>\mathrm{T}$ & p.(Leu1566Phe) & 21 & c.3056C $>$ T [26] & p.(Thr1019Met) & & & \\
\hline & & & 42 & c. $5882 \mathrm{G}>\mathrm{A}[25]$ & p.(Gly1961Glu) & & & & & & \\
\hline
\end{tabular}


Table 2. Cont

\begin{tabular}{|c|c|c|c|c|c|c|c|c|}
\hline CIC10754 & 5282 & $\begin{array}{l}\text { Unaffected } \\
\text { mother }\end{array}$ & & WILD TYPE & & 21 & c.3056C $>$ T [26] & p.(Thr1019Met) \\
\hline \multirow[t]{2}{*}{ CIC10755 } & 5282 & $\begin{array}{l}\text { Unaffected } \\
\text { father }\end{array}$ & 33 & c. $4696 \mathrm{C}>\mathrm{T}$ & p.(Leu1566Phe) & & WILD TYPE & \\
\hline & & & 42 & c.5882G>A [25] & p.(Gly1961Glu) & & & \\
\hline \multirow[t]{2}{*}{ CIC08932 } & 5089 & Index & 34 & c. $4775 \mathrm{G}>\mathrm{A}$ & p.(Gly1592Asp) & 3 & c. $288 \mathrm{C}>\mathrm{A}[47]$ & p.(Asn96Lys) \\
\hline & & & & & & 42 & c.5882G>A [25] & p.(Gly1961Glu) \\
\hline${ }^{++}$CIC08933 & 5089 & $\begin{array}{l}\text { Unaffected } \\
\text { father }\end{array}$ & & WILD TYPE & & & WILD TYPE & \\
\hline \multirow[t]{2}{*}{ CIC08934 } & 5089 & $\begin{array}{l}\text { Unaffected } \\
\text { mother }\end{array}$ & & WILD TYPE & & 3 & c. $288 \mathrm{C}>\mathrm{A}[47]$ & p.(Asn96Lys) \\
\hline & & & & & & 42 & c.5882G >A [25] & p.(Gly1961Glu) \\
\hline CIC07036 & 3867 & Index & 37 & c. $5282 C>G$ & p.(Pro1761Arg) & 42 & c.5882G>A [25] & p.(Gly1961Glu) \\
\hline \multirow[t]{2}{*}{ CIC07960 } & 4447 & Index & 37 & c. $5282 C>G$ & p.(Pro1761Arg) & 26 & c.3819dup [48] & p.(Leu1274Serfs*8) \\
\hline & & & 46 & c.6316C $>$ T [25] & p.(Arg2106Cys) & & & \\
\hline CIC07999 & 4447 & $\begin{array}{l}\text { Unaffected } \\
\text { sister }\end{array}$ & & WILD TYPE & & & WILD TYPE & \\
\hline \multirow[t]{2}{*}{ CIC08029 } & 4447 & $\begin{array}{l}\text { Unaffected } \\
\text { mother }\end{array}$ & 37 & c. $5282 C>G$ & p.(Pro1761Arg) & & WILD TYPE & \\
\hline & & & 46 & c.6316C $>$ T [25] & p.(Arg2106Cys) & & & \\
\hline \multirow[t]{2}{*}{ CIC09601 } & 5509 & Index & 37 & c. $5282 C>G$ & p.(Pro1761Arg) & 22 & c.3279C $>$ A [18] & p.(Asp1093Glu) \\
\hline & & & 46 & c. $6316 \mathrm{C}>\mathrm{T}[25]$ & p.(Arg2106Cys) & & & \\
\hline \multirow[t]{2}{*}{ CIC09602 } & 5509 & $\begin{array}{c}\text { Unaffected } \\
\text { daughter }\end{array}$ & 37 & c. $5282 C>G$ & p.(Pro1761Arg) & & WILD TYPE & \\
\hline & & & 46 & c.6316C > T [25] & p.(Arg2106Cys) & & & \\
\hline CIC07831 & 4373 & Index & 38 & c. $5332 \mathrm{~A}>\mathrm{T}$ & p.(Met1778Leu) & 27 & c.3899G >A [44] & p.(Arg1300Gln) \\
\hline CIC08439 & 4759 & Index & 38 & c. $5332 \mathrm{~A}>\mathrm{T}$ & p.(Met1778Leu) & 47 & c. $6394 \mathrm{G}>\mathrm{T}$ & p.(Glu2132 \\
\hline CIC08262 & 4633 & Index & 38 & c. $5342 \mathrm{C}>\mathrm{A}$ & p.(Ala1781Glu) & 3 & c. $286 \mathrm{~A}>\mathrm{G}$ [49] & p.(Asn96Asp) \\
\hline CIC08263 & 4633 & $\begin{array}{l}\text { Unaffected } \\
\text { mother }\end{array}$ & 38 & c. $5342 \mathrm{C}>\mathrm{A}$ & p.(Ala1781Glu) & & WILD TYPE & \\
\hline
\end{tabular}


Table 2. Cont

\begin{tabular}{|c|c|c|c|c|c|c|c|c|c|c|c|}
\hline CIC09095 & 4633 & $\begin{array}{l}\text { Unaffected } \\
\text { father }\end{array}$ & & WILD TYPE & & 3 & c. $286 \mathrm{~A}>\mathrm{G}$ [49] & p.(Asn96Asp) & & & \\
\hline CIC08359 & 4702 & Index & 38 & c. $5384 \mathrm{~T}>\mathrm{C}$ & p.(Leu1795Ser) & 17 & c.2588G >C [25] & p.(Gly863Ala) & 40 & c.5603A > T [34] & p.(Asn1868Ile) \\
\hline CIC07436 & 4110 & Index & IVS42 & c. $5898+2 \mathrm{~T}>\mathrm{C}$ & p.? & 40 & c.5642C>T [50] & p.(Ala1881Val) & & & \\
\hline CIC08523 & 4110 & $\begin{array}{l}\text { Unaffected } \\
\text { mother }\end{array}$ & & WILD TYPE & & 40 & c. $5642 \mathrm{C}>\mathrm{T}[50]$ & p.(Ala1881Val) & & & \\
\hline CIC08524 & 4110 & $\begin{array}{l}\text { Unaffected } \\
\text { father }\end{array}$ & IVS42 & c. $5898+2 \mathrm{~T}>\mathrm{C}$ & p.? & & WILD TYPE & & & & \\
\hline \multirow[t]{2}{*}{ CIC09857 } & 5675 & Index & 44 & c. $6110 \mathrm{C}>\mathrm{A}$ & p.(Ala2037Asp) & IVS38 & $\begin{array}{c}\text { c. } 5461-10 \mathrm{~T}>\mathrm{C} \\
{[45]}\end{array}$ & $\begin{array}{l}\text { p. } \\
\text { [Thr1821Valfs, } \\
\text { Thr1821Aspfs] }\end{array}$ & & & \\
\hline & & & & & & 40 & c.5603A>T [34] & p.(Asn1868Ile) & & & \\
\hline CIC09858 & 5675 & $\begin{array}{l}\text { Unaffected } \\
\text { mother }\end{array}$ & 44 & c. $6110 \mathrm{C}>\mathrm{A}$ & p.(Ala2037Asp) & & WILD TYPE & & & & \\
\hline \multirow[t]{2}{*}{ CIC10452 } & 5675 & $\begin{array}{l}\text { Unaffected } \\
\text { father }\end{array}$ & & WILD TYPE & & IVS38 & $\begin{array}{c}\text { c. } 5461-10 \mathrm{~T}>\mathrm{C} \\
{[45]}\end{array}$ & $\begin{array}{l}\text { p. } \\
\text { [Thr1821Valfs, } \\
\text { Thr1821Aspfs] }\end{array}$ & & & \\
\hline & & & & & & 40 & c.5603A>T [34] & p.(Asn1868Ile) & & & \\
\hline СIC01199 & 719 & Index & 45 & c.6181_6184del & p.(Thr2061Serfs*53) & 42 & c.5882G>A [25] & p.(Gly1961Glu) & & & \\
\hline CIC08054 & 719 & $\begin{array}{l}\text { Affected } \\
\text { sister }\end{array}$ & 45 & c.6181_6184del & p.(Thr2061Serfs*53) & 42 & c. $5882 \mathrm{G}>\mathrm{A}[25]$ & p.(Gly1961Glu) & & & \\
\hline CIC08057 & 719 & $\begin{array}{l}\text { Unaffected } \\
\text { father }\end{array}$ & & WILD TYPE & & 42 & c. $5882 \mathrm{G}>\mathrm{A}[25]$ & p.(Gly1961Glu) & & & \\
\hline CIC8058 & 719 & $\begin{array}{l}\text { Unaffected } \\
\text { mother }\end{array}$ & 45 & c.6181_6184del & p.(Thr2061Serfs*53) & & WILD TYPE & & & & \\
\hline \multirow[t]{2}{*}{ CIC03710 } & 1657 & Index & 45 & c.6191C >T & p.(Ala2064Val) & 30 & c.4537dup [44] & p.(Gln1513Profs & & & \\
\hline & & & 40 & c.5603A>T [34] & p.(Asn1868Ile) & & & & & & \\
\hline \multirow[t]{2}{*}{ CIC03711 } & 1657 & $\begin{array}{l}\text { Unaffected } \\
\text { mother }\end{array}$ & 45 & c. $6191 C>T$ & p.(Ala2064Val) & & WILD TYPE & & & & \\
\hline & & & 40 & c.5603A $>$ T [34] & p.(Asn1868Ile) & & & & & & \\
\hline \multirow[t]{2}{*}{ CIC04571 } & 2232 & Index & 45 & c. $6250 \mathrm{G}>\mathrm{A}$ & p.(Ala2084Thr) & 19 & c. $2894 \mathrm{~A}>\mathrm{G}[25]$ & p.(Asn965Ser) & & & \\
\hline & & & 42 & c.5882G>A [25] & p.(Gly1961Glu) & & & & & & \\
\hline
\end{tabular}


Table 2. Cont.

\begin{tabular}{|c|c|c|c|c|c|c|c|c|c|c|c|}
\hline CIC04572 & 2232 & $\begin{array}{l}\text { Unaffected } \\
\text { mother }\end{array}$ & & WILD TYPE & & 19 & c.2894A>G [25] & p.(Asn965Ser) & & & \\
\hline CIC 04573 & 2232 & $\begin{array}{c}\text { Unaffected } \\
\text { maternal } \\
\text { aunt }\end{array}$ & & WILD TYPE & & 19 & c.2894A>G [25] & p.(Asn965Ser) & & & \\
\hline \multirow[t]{2}{*}{ CIC07568 } & 2232 & $\begin{array}{l}\text { Unaffected } \\
\text { father }\end{array}$ & 45 & c. $6250 \mathrm{G}>\mathrm{A}$ & p.(Ala2084Thr) & & WILD TYPE & & & & \\
\hline & & & 42 & c.5882G $>$ A [25] & p.(Gly1961Glu) & & & & & & \\
\hline CIC07748 & 4312 & Index & 46 & c. $6284 A>G$ & p.(Asp2095Gly) & 22 & c.3323G>A [34] & p.(Arg1108His) & & & \\
\hline CIC09593 & 4312 & $\begin{array}{l}\text { Unaffected } \\
\text { mother }\end{array}$ & 46 & c. $6284 \mathrm{~A}>\mathrm{G}$ & p.(Asp2095Gly) & & WILD TYPE & & & & \\
\hline CIC09594 & 4312 & $\begin{array}{l}\text { Unaffected } \\
\text { father }\end{array}$ & & WILD TYPE & & 22 & c.3323G $>$ A [34] & p.(Arg1108His) & & & \\
\hline CIC09374 & 5379 & Index & 47 & c. $6455 \mathrm{G}>\mathrm{T}$ & p.(Gly2152Val) & 9 & c. $1222 \mathrm{C}>\mathrm{T}[43]$ & p. $\left(\operatorname{Arg} 408^{*}\right)$ & & & \\
\hline CIC09375 & 5379 & $\begin{array}{l}\text { Unaffected } \\
\text { mother }\end{array}$ & 47 & c. $6455 \mathrm{G}>\mathrm{T}$ & p.(Gly2152Val) & & WILD TYPE & & & & \\
\hline CIC06396 & 3389 & Index & 47 & c.6436_6437insT & p.(Gly2146Valfs*36) & 47 & c.6436_6437insT & $\begin{array}{c}\text { p. }( \\
\text { Gly2146Valfs*36) }\end{array}$ & & & \\
\hline CIC00130 & 102 & Index & 48 & c.6693del & p.(Ile2231Metfs*16) & 44 & c.6079C $>$ T [25] & p.(Leu2027Phe) & & & \\
\hline CIC00131 & 102 & $\begin{array}{l}\text { Unaffected } \\
\text { father }\end{array}$ & 48 & c.6693del & p.(Ile2231Metfs*16) & & WILD TYPE & & & & \\
\hline CIC00132 & 102 & $\begin{array}{l}\text { Unaffected } \\
\text { mother }\end{array}$ & & WILD TYPE & & 44 & c. $6079 \mathrm{C}>\mathrm{T}[25]$ & p.(Leu2027Phe) & & & \\
\hline CIC09114 & 5205 & Index & 48 & c. $6704 \mathrm{C}>\mathrm{G}$ & p.(Ser2235*) & 21 & c.3113C > T [24] & p.(Ala1038Val) & & & \\
\hline CIC08581 & 4855 & Index & 49 & c. $6746 \mathrm{C}>\mathrm{A}$ & p.(Ala2249Asp) & 13 & c.1819G>A [40] & p.(Gly607Arg) & 42 & c.5882G $>$ A [25] & p.(Gly1961Glu) \\
\hline CIC08381 & 4722 & Index & IVS49 & c. $6816+1 G>T$ & p.? & 14 & c. 2023G >A [38] & p.(Val675Ile) & & & \\
\hline CIC08383 & 4722 & $\begin{array}{l}\text { Affected } \\
\text { sister }\end{array}$ & IVS49 & c. $6816+1 G>T$ & p.? & 14 & c. $2023 \mathrm{G}>\mathrm{A}[38]$ & p.(Val675Ile) & & & \\
\hline CIC08402 & 4722 & $\begin{array}{l}\text { Unaffected } \\
\text { mother }\end{array}$ & & WILD TYPE & & 14 & c.2023G>A [38] & p.(Val675Ile) & & & \\
\hline
\end{tabular}

${ }^{+}$: CIC03678 is carrier of a variant of uncertain significance (c.2868C >A), and hence was excluded from genotype-phenotype analysis. ${ }^{++}$: CIC08933, unaffected father of CIC08932 does not carry any likely pathogenic variant therefore variant c.4775G $>$ A could be de novo or CIC08933 is not the biological father of CIC08932. p.?: Protein has not been analysed, an effect is expected but difficult to predict. 
All novel variants found in the study and their respective position on $A B C A 4$ are represented in Figure 2.

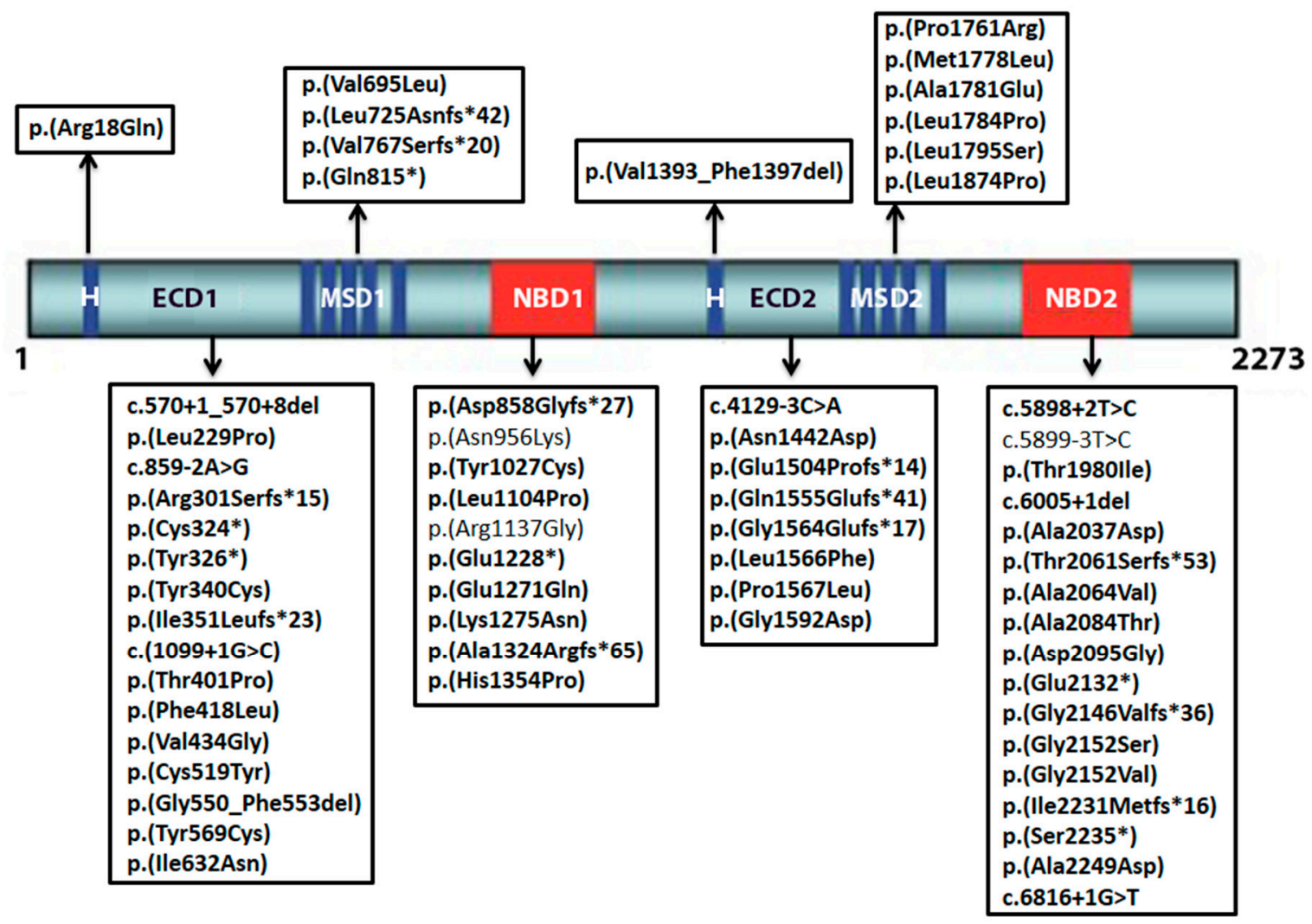

Figure 2. Location of novel $A B C A 4$ variants identified in the study. Nucleotide positions and translation correspond to CCDS747.1 and NP_000341.2, respectively. Only the three mutations of uncertain significance are not in bold letters; ECD1: first extracellular domain; NBD1: first nucleotide binding domain; MSD1: first membrane spanning domain; H: hydrophobic domain; ECD2: second extracellular domain; NBD2: second nucleotide binding domain; MSD2: second membrane spanning domain.

\subsection{Genotype-Phenotype Correlation}

Genotype-phenotype correlation analysis was performed on the 60 patients carrying at least one likely pathogenic variant on each allele. The three subjects carrying a VUS were excluded from the analysis. All clinical features are presented in the Supplementary Table S3.

Patients were divided in two genotype groups: (1) patients with at least one null mutation (NM), i.e., frame-shift or nonsense mutations, splicing variants affecting the first two nucleotides adjacent to an exonic sequence and/or other variants proven to cause a premature stop or incomplete formation of the whole protein in previous studies (e.g., c.5461-10T>C [51]); (2) patients with two or more missense variants (MM) [52].

The two genotypic groups were not statistically different for age (mean of $34.3 \pm 17.8$ years for NM group and $33.9 \pm 18.3$ years for MM group; $p=0.94$ ) nor duration of disease (mean of $16.9 \pm 14.6$ years for NM group and $10.8 \pm 10.9$ years for MM group; $p=0.095$ ).

Mean age of onset was $18.5 \pm 12.59$ years and $23.1 \pm 14.8$ years in NM and MM groups, respectively $(p=0.037)$.

Right eye (RE) and left eye (LE) did not differ significantly for best corrected visual acuity (BCVA) $(p=0.81)$, central retinal thickness (CRT) $(p=0.09)$, and macular volume (MV) ( $p=0.137)$, therefore we chose to use data from the right eye for comparison between genotypic groups. 
Null mutation and MM groups did not differ significantly for optical coherence tomography (OCT) parameters: mean CRT was $126.7 \pm 45.3 \mu \mathrm{m}$ and $122 \pm 26.1 \mu \mathrm{m}$, respectively $(p=0.89)$, while mean MV was $6.2 \pm 1.3 \mathrm{~mm}^{2}$ and $6.8 \pm 1.1 \mathrm{~mm}^{2}$, respectively ( $p=0.13$ for MV). Mean BCVA was $0.97 \pm 0.48 \log$ MAR in the NM group and $0.85 \pm 0.29 \log$ MAR in the MM group $(p=0.26)$ (Figure 3 )
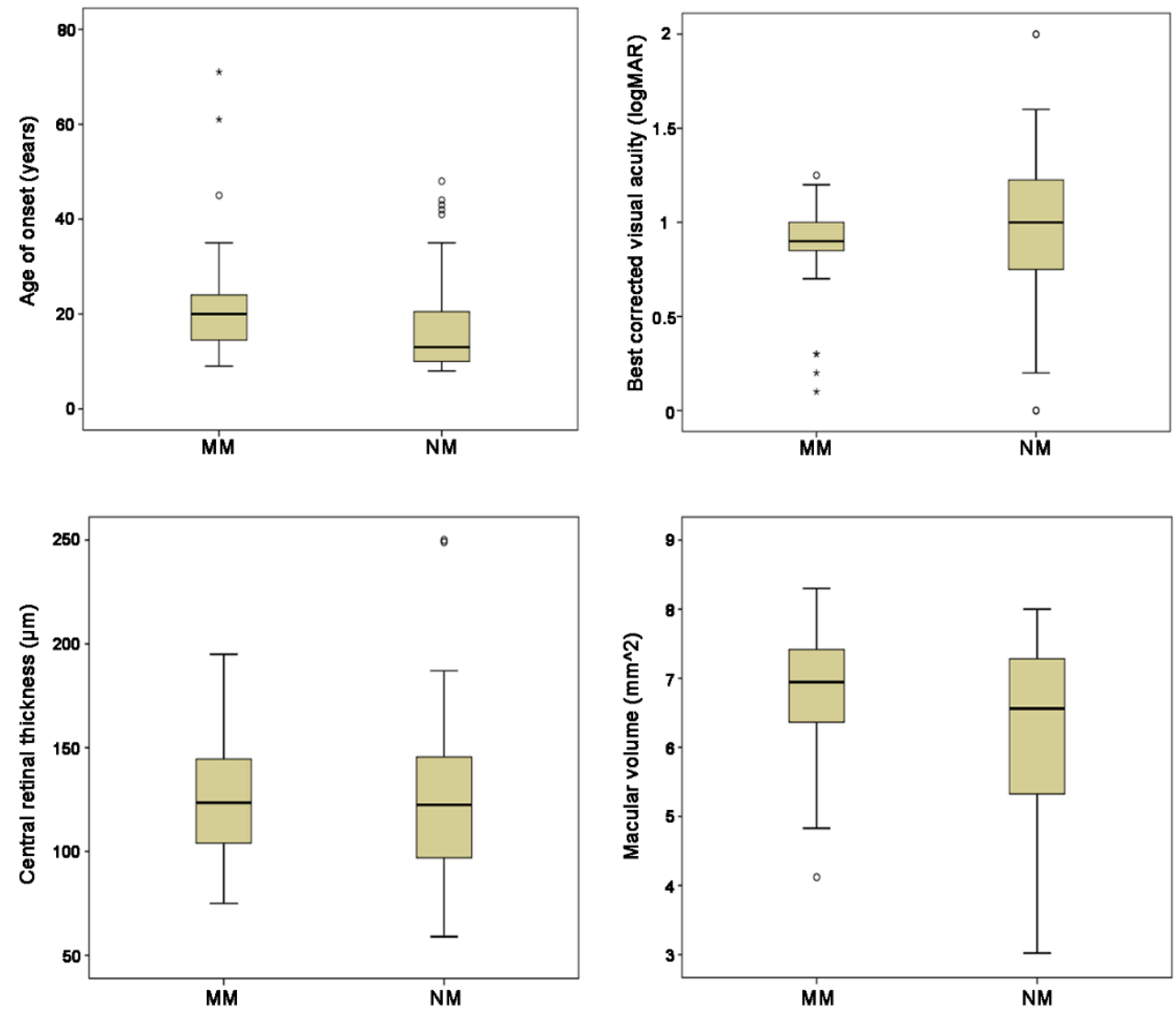

Figure 3. Box-plots showing the comparison of age of onset (AO), best corrected visual acuity (BCVA), central retinal thickness (CRT), and macular volume (MV) for the two genotype groups (patients with at least one null mutation (NM) and patients with two or more missense variants (MM)). The dark line in the middle of the boxes is the median; the bottom of the box indicates the 25th percentile while the top of the box represents the 75th percentile. The T-bars that extend from the boxes represent the minimum and maximum values when they are within 1.5 times the height of the box. The circles are outliers (i.e., values that do not fall in the T-bars). The stars are extreme outliers (i.e., values more than three times the height of the boxes). Only AO shows a statistically significant difference between NM and MM.

The phenotype was classified following all clinical criteria summarized in Table 3. 
Table 3. Clinical criteria used to classify patients in our study. FP: fundus photography; SW-AF: short-wavelength fundus autofluorescence; NIR-AF: near-infrared fundus autofluorescence; ERG: electroretinogram; OCT: optical coherence tomography; RPE: retinal pigment epithelium; EZ: ellipsoid zone; FS: foveal sparing.

\begin{tabular}{|c|c|c|c|}
\hline & Groups/Stages & Criteria & Reference \\
\hline Age of onset & n.a. & Age at which visual loss was first noticed & Lois et al., 2001 [53] \\
\hline \multirow{4}{*}{ FP } & Stage 1 & Central macular atrophy with parafoveal or perifoveal flecks & \multirow[t]{4}{*}{ Fishman GA et al., 1976 [54] } \\
\hline & Stage 2 & Numerous flecks extended anterior to the vascular arcades and/or nasal to the optic disc & \\
\hline & Stage 3 & Desorbed flecks with choriocapillaris atrophy within the macula & \\
\hline & Stage 4 & Widespread RPE and chorioretinal atrophy throughout the fundus defined stage & \\
\hline \multirow{8}{*}{ SW-AF and NIR-AF } & Group 1 & Central lesion with jagged border & \multirow[t]{5}{*}{ Duncker et al., 2014 [55] } \\
\hline & Group 2 & Lesion with extensive fundus changes & \\
\hline & Group 3 & Central lesion with smooth border and hyperautofluorescent SW-AF and NIR-AF ring & \\
\hline & Group 4 & Central lesion with smooth border and no hyperautofluorescent NIR-AF ring & \\
\hline & Group 5 & Discrete central lesions better visualized in NIR-AF images & \\
\hline & Peripapillary area preserved & No alterations within an eccentricity of $0.6 \mathrm{~mm}$ from the optic disc & \multirow{3}{*}{ Cideciyan et al., 2005 [56] } \\
\hline & Flecks in the peripapillary area & Presence of flecks within an eccentricity of $0.6 \mathrm{~mm}$ from the optic disc & \\
\hline & Peripapillary area not preserved & Absence of EZ band and/or EPR atrophy within an eccentricity of $0.6 \mathrm{~mm}$ from the optic disc & \\
\hline \multirow{3}{*}{ ERG } & I & Normal scotopic and full-field ERG & \multirow[t]{3}{*}{ Lois et al., 2001 [53] } \\
\hline & II & Loss of photopic function & \\
\hline & III & Loss of both photopic and scotopic function & \\
\hline \multirow{2}{*}{ SW-AF and OCT } & FS-YES & Foveal sparing & \multirow[t]{2}{*}{ Fujinami et al., 2013 [38] } \\
\hline & FS-NO & Early onset foveal atrophy & \\
\hline \multirow{3}{*}{ OCT } & EZ Absent & EZ band loss & \multirow[t]{3}{*}{ Parodi et al., 2015 [57] } \\
\hline & EZ Disrupted & EZ band disorganization & \\
\hline & EZ Preserved & Identification of EZ band & \\
\hline \multirow{2}{*}{ Genotype } & NM & At least one null or splice variant is present & \multirow[t]{2}{*}{ Fujinami et al., 2013 [52] } \\
\hline & MM & Two or more missense variants are present & \\
\hline
\end{tabular}


Fundus staging, short-wavelength fundus autofluorescence (SW-FAF), and near infrared fundus autofluorescence (IR-FAF) groups, foveal sparing, the presence of retinal pigment epithelium (RPE) atrophy and the integrity of the ellipsoid zone (EZ) band were not statistically different between the $\mathrm{NM}$ and MM groups ( $p=0.315, p=0.855, p=0.441, p=0.327, p=0.089$, respectively). However, electroretinogram (ERG) group distribution and the presence of autofluorescence peripapillary sparing significantly differed between the two groups ( $p=0.002$ and $p=0.003$ respectively) (Figure 4 ).
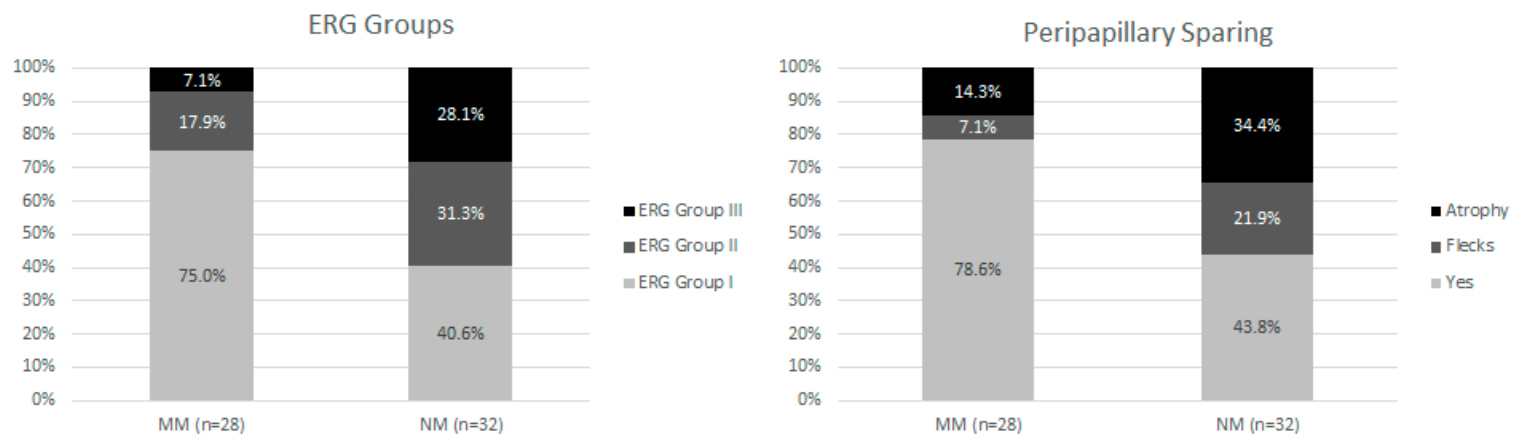

Figure 4. Repartition between the two genotypic subtypes for electroretinography (ERG) groups (on the left) and peripapillary sparing (on the right). For ERG classification, group I has abnormal multifocal (mf-) ERG with normal full-field (ff-) ERG; in group II there were mf-ERG abnormalities with cone dysfunction (assessed with light-adapted $30 \mathrm{~Hz}$ flicker and light-adapted 3.0); Group III has additional rod dysfunction (assessed using dark-adapted 0.01 and dark-adapted 10.0). Peripapillary area was considered speared (Yes in the graph) if no alterations were found in the fundus autofluorescence within $0.6 \mathrm{~mm}$ from the optic disc edge. This area was not considered speared when flecks or ellipsoid zone (EZ) and/or retinal pigment epithelium (RPE) atrophy were present (see Table 3 for classifications). Photoreceptors dysfunction (ERG groups II and III) and peripapillary area involved with the presence of flecks or atrophy are more prevalent in the group of patients with at least one null mutation.

\section{Discussion}

This work is a longitudinal study starting in 2007 which used a combination of microarray analysis and direct Sanger sequencing to identify $A B C A 4$ mutations in a large French cohort with a clinical diagnosis of STGD1 disease. At that time targeted NGS and whole exome sequencing (WES) was not available in our laboratory. The rate of bi-allelic variants detected in the population using this approach was $75.6 \%$, which is in accordance with previously reported data (about 75\%) [15-19].

The authors believe that the same results would have been obtained using only direct Sanger sequencing on the entire cohort. Initially, patients were analyzed with microarray to evaluate the prevalence of known mutations, which was at this time commonly used to reduce screening costs. However, the high number of unsolved cases led us to further investigate the cohort with direct Sanger sequencing. The sole application of microarray was enough to solve 76 cases, which were not further investigated with Sanger technique. Indeed, this may have led to an underestimation of the prevalence of novel additional mutations and/or complex alleles in this cohort $[28,58]$. The relatively high number of unsolved cases may be related to a combination of different factors: (1) The clinical overlap of distinct genetic entities could have led to uncertainties in patients' selection. Therefore the screening of other genes (e.g., ELOVL4, PROM1, PRPH2, etc.) responsible for overlapping phenotypes may help solving such cases; (2) Large rearrangements within the $A B C A 4$ locus have been shown to occur in $\sim 0.5 \%$ to $\sim 2 \%$ of STGD1 cases in previous reports and they would not have been detected by our mutational screening [22,24]; (3) Mutations in noncoding regions of the $A B C A 4$ gene locus have been proposed as a common source for a second causative mutation in patients with typical Stargardt phenotype. In particular, the occurrence of deep intronic variants in subjects with a single $A B C A 4$ 
mutations may range from $\sim 2 \%$ to $\sim 18 \%$ in different reports [19-22,27,59]. This may have led not only to an underestimation of bi-allelic cases, but also of the number of novel variants found in the cohort, whose report was the main aim of this study. However, the number of novel variants affecting deep intronic regions should be relatively small. Schulz et al. [27] in a recent report screened for deep intronic variants 237 STGD1 patients showing a single $A B C A 4$ variant. Among the cohort, ten different sequence variants were found, only two of which were not previously reported [27]. The addition of the first, the screening of selected noncoding regions known to be "hot spots" for pathogenic variants (e.g., intron 30 and 36 [27]), and second, whole genome sequencing (WGS) analysis, will be the next steps in order to raise the number of solved cases in our cohort.

Among the 305 patients with bi-allelic variants, we found a total of 240 disease-causing mutations, of which $60(25 \%)$ were never described before (Table 2).

The high number of new variants found in this cohort may seem surprising considering that several studies had already been conducted on the western and central European populations $[5,15,17,27,43]$. These findings are probably related to the extensive allelic heterogeneity of $A B C A 4$ and the possible contribution of ethnic differences [12]. Among the 61 patients with novel likely pathogenic or pathogenic variants, three subjects had eastern European origins and nine subjects had non-European origins. The novel variant p.(Pro1761Arg) is carried by three subjects with no ties of consanguinity and with non-European origins: Armenia (CIC07960), Turkey (CIC09601), and Algeria (CIC07036). This may suggest a higher prevalence of this mutation in the south Mediterranean area. Both CIC07960 and CIC09601 harbor the variant in cis with p.(Arg2106Cys). Interestingly, the two subjects share the same pattern distribution of the lesion, but CIC07960 has an earlier age of onset and an important foveal involvement, probably due to the associated truncating mutation p.(Leu1274Serfs*8) (Figure 5).

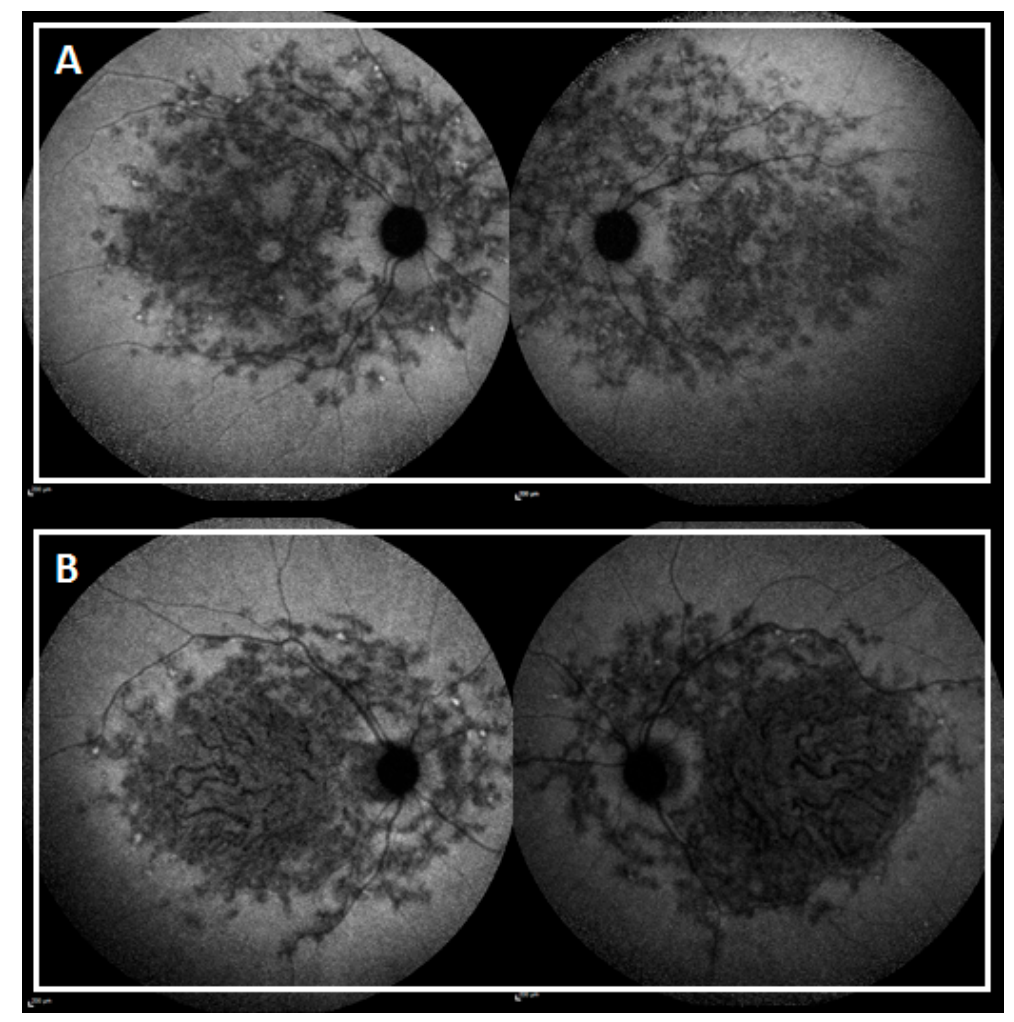

Figure 5. Near infrared autofluorescence (SW-AF) of subjects CIC09601 (A) and CIC07960 (B).

The novel variant p.(Ile351Leufs*23) was carried by three unrelated French subjects indicating a possible higher prevalence in this population. While CIC01080 and CIC04235 share an early onset 
advanced disease with spread fundus atrophy, CIC07308 has a normal ff-ERG and no posterior atrophy probably due to the second mutation p.(Gly1961Glu), known for its "milder" pathologic effect [30,31].

Among the likely pathogenic novel variants three were homozygous: p.(Tyr340Cys), p.(Val434Gly), and p.(Gly2146Valfs*36).

CIC07725, harboring p.(Tyr340Cys), had early-onset disease (nine years of age) with normal ff-ERG, centripetal spread of flecks and foveal EZ band involvement. CIC09625, harboring p.(Val434Gly) has an early onset disease (nine years of age) with a central lesion without flecks and with foveal sparing. Even though they share a similar age of onset and duration of the disease (one and two years, respectively), CIC09625 shows a "milder" phenotype (Figure 6). These two missense mutations may have a distinct impact on the protein function even though they are located in the same domain (first ABCA4 extracellular domain). Alternatively, genetic or environmental modifiers may explain the phenotypic variability.

As expected, CIC06396, homozygous for p.(Gly2146Valfs*36), showed a more advanced phenotype with an early onset disease (nine years of age), extensive fundus RPE atrophy and photoreceptor impairment (Figure 6).

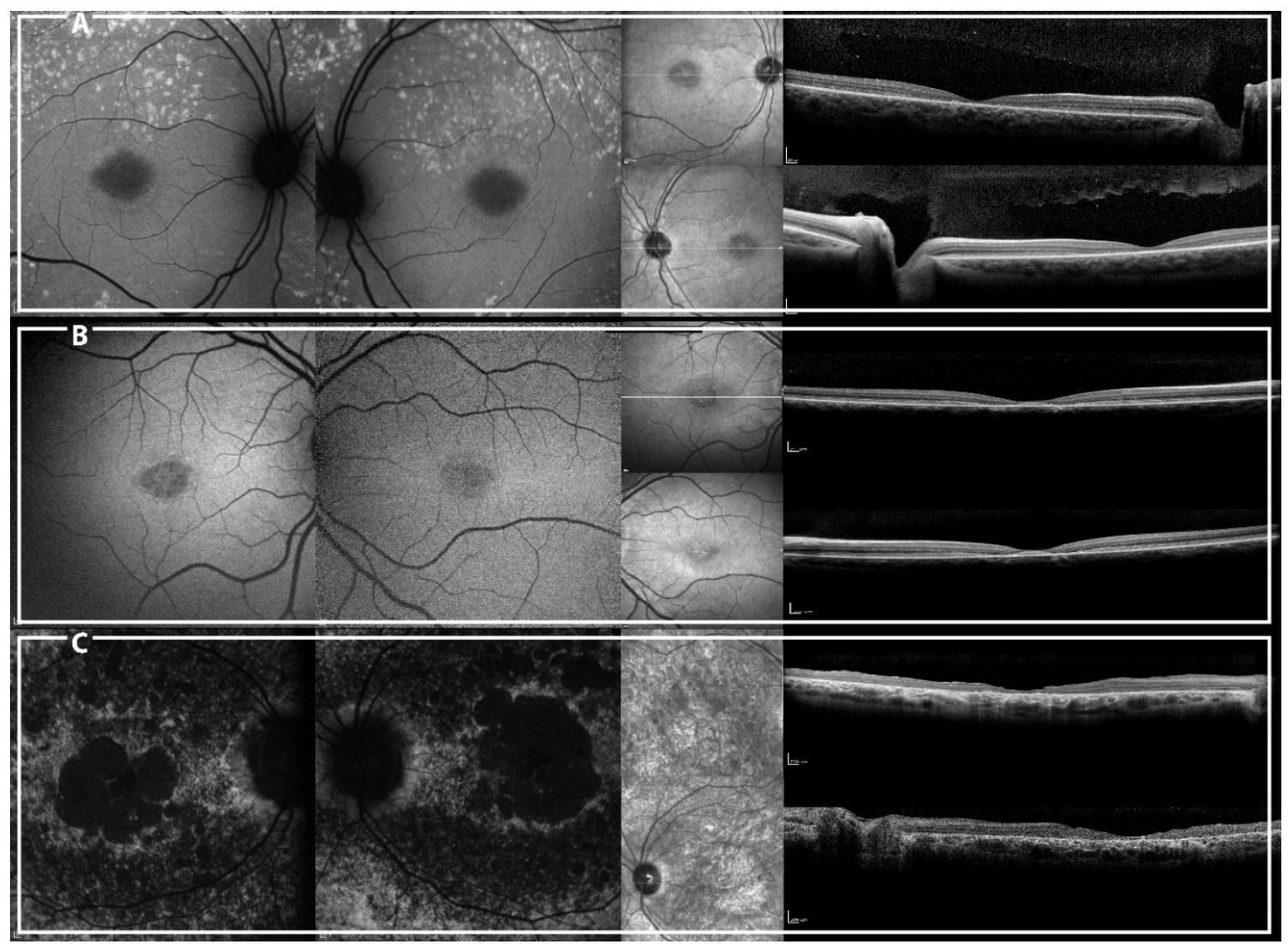

Figure 6. Short-wavelength autofluorescence (SW-AF) (left) and spectral domain optical coherence tomography (SD-OCT) central line (right) of patients CIC07725 (A), CIC09625 (B), and CIC06396 (C).

VUS identified in the cohort were two missense variants: p.(Asn956Lys), p.(Arg1137Gly), and one non-canonical splice-site variant (c.5899-3T>C) (Table 1 and Supplementary Table S2).

CIC05824 harbors the novel intronic variant c.5899-3T>C, which is not predicted to affect splicing by the algorithms used since the nucleotide is poorly evolutionarily conserved (Supplementary Table S2). Indeed, we cannot exclude the possibility that this variant may affect splicing in vivo since it has already been proven that $A B C A 4$ can have non-canonical splice sites variant with important effects on protein [19-23,59]. 
The VUS p.(Asn956Lys) harbored by CIC03678 is predicted to be pathogenic only by SIFT and the AA is not conserved (Table 1, Supplementary Table S2). Asparagine and Lysine share similar characteristics of hydropathy and polarity. Unfortunately, although genetic analysis of CIC03679, unaffected son of CIC03678, revealed that he was not carrying p.(Asn956Lys), in absence of other family members available for co-segregation analysis, its significance still remains unclear.

Variant c.3409A $>$ G, p.(Arg1137Gly) carried by CIC03545, is predicted to be pathogenic by SIFT and mutation taster although the residue was not conserved, being substituted by a Glutamine, a Threonine or a Lysine in the conservation analysis (Table 1, Supplementary Table S2). However, all these amino acids are polar while Glycine is not, hence an effect on the tridimensional structure of the protein cannot be completely excluded.

Several hypomorphic alleles have been associated with STGD1 [27,29]. Even though many of them were considered benign because of their high frequency, these alleles could be pathogenic when they are in trans with severe variants, explaining many of the unsolved cases with only one or no mutations detected on $A B C A 4[27,29]$. The presence of these alleles could help in classifying accompanying $A B C A 4$ mutations as severe mutations and in genotype-phenotype correlations.

For example, Zernant et al. [29] recently reported that the hypormophic variant p.(Asn1868Ile) accounts for more than $50 \%$ of the missing causal alleles in monoallelic cases and about $80 \%$ of late-onset cases and distinguishes $A B C A 4$-related disease from AMD [29]. In our sub-cohort of 61 patients, this variant appeared in 14 subjects. Most of these cases presented p.(Asn1868Ile) in cis with other disease causing mutations (12 subjects) and the phenotypes were typically consistent with the effect of the overall genotype with mainly early onset and severe disease as documented by Zernant et al. [29]. Only two subjects (CIC08283 and CIC04197) had p.(Asn1868Ile) alone, in trans with other pathogenic variants, resulting in a milder phenotype. Further functional analysis should be performed in order to confirm the pathogenicity of these variants.

Among the 63 patients carrying novel variants, we performed genotype-phenotype correlation on 60 of them. The three subjects carrying a VUS were excluded from the analysis. According to the genotype-phenotype model previously proposed [24], we observed an earlier age of onset and more diffuse photoreceptor dysfunction (assessed by ff-ERGs) in NM group. These findings are in accordance with the previous literature [12,52]. Regarding the peripapillary sparing, its loss was more frequent in the NM group which is also associated with a higher prevalence of non-group I ff-ERG consistent with previous publications reporting a loss of this sign in non-group I Stargardt disease [60].

Overall, in our group of 60 patients harboring novel likely pathogenic $A B C A 4$ changes, we observed a tendency for a worse phenotype when a truncating variant is present.

In this report we wanted to prioritize the characterization of the patients carrying novel variants. Even though it is only a subgroup of subjects the results seem strong and consistent with previous literature [12] and they probably reflect the genotype-phenotype correlation of the entire cohort. Further analysis including a full clinical assessment of patients with known variants will be necessary to confirm these findings.

However, even with a large cohort of patients, it is impossible to establish a precise phenotype-genotype correlation only on the basis of the type of the mutation. In fact, two different missense mutations involving residues at different location with distinct effects on the quaternary structure of ABCA4 can produce very different phenotypes [12,61]. Nowadays, the main efforts are aimed to find correlations between specific variants and clinical phenotype although the rarity of many alleles makes this difficult. For this reason, we tried to provide an exhaustive clinical characterization of each subject showing at least one novel mutation among the two mutant alleles of ABCA4 (see Supplementary Table S3). 


\section{Materials and Methods}

Patients with a presumed diagnosis of STGD1 disease were recruited at the Reference Center for rare diseases, Referet of the Quinze-Vingts hospital, Paris. Informed consent was obtained from each patient after explanation of the study and its potential outcome. The study protocol adhered to the tenets of the Declaration of Helsinki and was approved by a national ethics committee (CPP Ile de France V, Project number 06693, NoEUDRACT 2006-A00347-44, 11 December 2006). All the patients and available family members were asked to donate a blood sample for genetic screening for $A B C A 4$ mutations. Subjects with at least one novel variant were called back to undergo a complete phenotype analysis as described below.

\subsection{Mutation Analysis}

Total genomic DNA was extracted from peripheral whole blood samples by standard salting out procedures according to the manufacturer's recommendation (Puregen Kit; Qiagen, Courtaboeuf, France). The first consecutive 211 subjects were screened for known $A B C A 4$ mutations by microarray analysis on a commercially available microarray (ABCR600, ASPER Biotech, Inc., Tartu, Estonia) [48]. Among them, samples which were excluded for known variants were further investigated for variants in the coding exons and their flanking regions of $A B C A 4$ by PCR and direct Sanger sequencing. The DNA of the other patients included in the study was directly sequenced by Sanger sequencing. The 50 coding exons and flanking intronic regions were amplified in 48 fragments ( $A B C A 4$ RefSeq NM_000350) using oligonucleotides reported in Supplementary Table S4, a commercially available polymerase (HotFire, Solis Biodyne, Tartu, Estonia), $1.5 \mathrm{mM} \mathrm{MgCl}_{2}$ at an annealing temperature of $58^{\circ} \mathrm{C}$ for $1 \mathrm{~min}$. The PCR products were enzymatically purified (ExoSAP-IT, USB Corporation, Cleveland, $\mathrm{OH}, \mathrm{USA}$, purchased from GE Healthcare, Orsay, France) sequenced and investigated as previously reported [62].

Nucleotide numbering reflects cDNA numbering with +1 corresponding to the A of the ATG translation initiation codon in the reference sequence, according to journal guidelines (www.hgvs.org/ mutnomen).

All variants were classified following the American College of Medical Genetics and Genomics (ACMG) standards and guidelines and the Association of Molecular Pathology (AMP) Clinical Practice Guidelines and Reports based on previous publications (as compiled in the Human Gene Mutation Database (HGMD) [63] and in the Leiden Open (source) Variation Database (LOVD) [64]), population data, computational data and functional data. The Exome Aggregation Consortium (ExAC) database was used to check variant frequency.

Novel variants with unknown frequency or minor allele frequency (MAF) $\leq 0.05$ were further tested using Alamut Visual software v. 2.7.1 where the in silico predictive programs PolyPhen2 (Polymorphism Phenotyping, http:/ / genetics.bwh.harvard.edu/pph2/) [65], SIFT (Sorting Intolerant from Tolerant; http://sift.bii.a-star.edu.sg/) [66], Mutation Taster (http://www.mutationtaster. org /) [67] are implemented.

For splice site variants three different algorithms of prediction were used: MaxEntScan (http: / / genes.mit.edu/burgelab/maxent/Xmaxentscan_scoreseq.html) [68], NNSplice [69], and human splicing finder v 3.0 (http:/ / www.umd.be/HSF3/) [70].

Evolutionary conservation was investigated using the 46-way Vertebrate Multiz Alignment and Conservation of the University of California Santa Cruz (UCSC) genome browser [71,72].

For missense mutations, an amino acid residue was considered highly conserved if the same residue was present in all species or was different in just one species among fishes or reptiles, moderately conserved if different in 2 to 5 species (included), and not conserved if different in more than 5 species or in at least one primate.

A novel sequence variant was considered pathogenic if it represented a nonsense variant or small insertion, deletion, or duplication inducing a frame-shift. In the case of a missense change, a novel sequence variant was considered to be likely pathogenic if it was either predicted to be deleterious by 
all three prediction algorithms or if it affected a highly or moderately evolutionary conserved amino acid residue and was predicted to be pathogenic by at least one algorithm mentioned above [73]. In all other cases, a variant was classified as a VUS.

The same criteria described above, but considering DNA sequence, were applied to study the conservation of nucleotide residues and pathogenicity of variants on putative or non-canonic splice sites ( \pm 10 bases from exon boundaries).

\subsection{Phenotypic Analysis}

Patients with novel $A B C A 4$ variants underwent full ophthalmic examination with assessment of best corrected visual acuity (BCVA) with the Early Treatment Diabetic Retinopathy Study (ETDRS) chart, kinetic and static perimetry, and color vision with the desaturated Farnsworth Panel D-15. Full-field and multifocal electroretinography (ff-ERG and mf-ERG) were performed with DTL recording electrodes and incorporated ISCEV Standards (Espion E2; for full field ERG; Diagnosys, Lowell, MA, USA; and Veris II for multifocal ERG; EDI, Redwood City, CA, USA) [74,75]. Clinical assessment was completed with color fundus photograph (FP; Topcon, Tokyo, Japan), short-wavelength fundus autofluorescence (SW-AF), near-infrared fundus autofluorescence (NIR-AF), spectral domain optical coherence tomography (SD-OCT; Heidelberg retina angiograph [HRA] II or Spectralis HRA+OCT; Heidelberg Engineering, Dossenheim, Germany) after pupil dilatation with Tropicamide $1 \%$ and phenylephrine $2.5 \%$.

The phenotype was classified following all clinical criteria summarized in Table 3. Color fundus photographs were acquired for each index patient and stratified in 4 groups according to a previously described classification [54].

A confocal scanning laser ophthalmoscope (Heidelberg Retina Angiograph [HRA] II; Heidelberg Engineering, Dossenheim, Germany) was used to acquire SW-AF images (488 nm excitation) and NIR-AF images (787 $\mathrm{nm}$ excitation). Thanks to the eye-tracking function of the HRA and averaging at least 30 single frames, 2 high-quality images $\left(30^{\circ}\right.$ and $50^{\circ}$ field of view respectively) for each modality were taken for each eye. Subsequently, we divided patients into 5 groups according to a previously described classification [55]: group 1: central lesion with jagged borders, group 2: central lesion with extensive fundus changes; group 3: central lesion with smooth borders and an hyperautofluorescent ring-like halo in SW-AF and NIR-AF; group 4: central lesion with smooth borders and no hyperautofluorescent NIR-AF ring; group 5: small discrete central lesion better visualized in NIR-AF. When RPE atrophy was present the measurement of the area was performed using RegionFinder software (Heidelberg Engineering, Dossenheim, Germany; software version 2.5.8.0) on SW-AF images [76]. The $50^{\circ}$ images were used to establish the extension of fundus abnormalities and for evaluating the peripapillary area [56].

A single horizontal high-resolution OCT B-Scan $(9 \mathrm{~mm})$ was obtained together with a simultaneously acquired infra-red (IR) fundus image in a transverse plane through the fovea. This image was used to evaluate the preservation of the ellipsoid zone (EZ) through the fovea [57] and the possible foveal sparing from the disease [38]. A cube of OCT scans was then obtained in high-speed mode with the automated real-time mode activated and set to 10 (creating a mean image of ten repeated identical B-scans to improve signal-to-noise ratio). The cube scan protocol contains a density of $49 \mathrm{~B}$-scan lines covering an area of $20^{\circ} \times 20^{\circ}$ centered on the fovea. As previously described, a trained operator corrected any segmentation errors made by the software version with manual segmentation $[77,78]$. When the quality of segmentation was adequate, the central retinal thickness (CRT) and the total macular volume (TMV) were recorded and compared against the data of 30 normal subjects ( 15 subjects younger than 30 years and 15 subjects older; Supplementary Table S5).

All patients underwent electrophysiological assessment, including ff-ERG and mf-ERG, incorporating the minimum standards of the International Society for Clinical of Electrophysiology of Vision (ISCEV) including the following stimulations: dark adapted dim flash 0.01 candela second $\left(\mathrm{cd} \cdot \mathrm{s} / \mathrm{m}^{2}\right)$; dark-adapted bright flash $10.0 \mathrm{~cd} \cdot \mathrm{s} / \mathrm{m}^{2}$; light adapted $3.0 \mathrm{~cd} \cdot \mathrm{s} / \mathrm{m}^{2} 30 \mathrm{~Hz}$ flicker 
ERG and light adapted $3.0 \mathrm{~cd} \cdot \mathrm{s} / \mathrm{m}^{2}$ at $2 \mathrm{~Hz}$. The patient data set were compared against those of 30 healthy subjects (15 younger than 30 years old and 15 older; Supplementary Table S1). The limits of ERG normality were defined for all the components of the ERG as the mean value \pm 2 standard deviations. All the components of the ERG from each eye were taken into account when classifying patients into the three ERG groups defined by Lois et al. [53]: Group 1 has abnormal mf-ERG with normal ff-ERG; in Group 2 there were mf-ERG abnormalities with cone dysfunction (assessed with light-adapted $30 \mathrm{~Hz}$ flicker and light-adapted 3.0); Group 3 has additional rod dysfunction (assessed using dark-adapted 0.01 and dark-adapted 10.0). The overall classification was based on the more severe eye when the ERG group was different between eyes in the same patient.

\subsection{Statistical Analysis}

All data analysis was conducted using IBM SPSS Statistics software v. 21.0 (Chicago, IL, USA).

For BCVA, MV, and CRT we used the Wilcoxon signed-rank test to prove the agreement between eyes.

Differences between the two genotype groups were analyzed using the Wilcoxon-Mann-Whitney test for age of onset, age at visit, duration of the disease, BCVA, MV, and CRT. The Goodman-Kruskal Gamma test was used to analyze the difference between NM and MM groups for fundus stage, NIR-FAF, and IR-FAF groups, the presence of RPE atrophy, foveal sparing, peripapillary sparing, EZ band integrity, and ERG group distribution. For these descriptive variants, when there was discordance between the right and left eye, we considered the eye with the worse phenotype. A $p$ value inferior to 0.05 was considered statistically significant.

\section{Conclusions}

Despite certain limitations in terms of detecting large CNVs $(<1 \%$ of all disease-associated alleles) [79] or mutations in non-coding regions, and the lack of functional analysis, our study broadens the spectrum of $A B C A 4$ mutations with 60 likely pathogenic or pathogenic variants, all associated with STGD1.

Supplementary Materials: Supplementary materials can be found at http:/ /www.mdpi.com/1422-0067/19/8/ 2196/s1.

Author Contributions: M.N., C.Z., and I.A. were involved in study design, collection and analysis of the data as well as drafting of the manuscript. A.A., S.M.-S., C.-M.D., C.M., C.M.E., and J.-A.S. were involved in study design, data collection, and critical review of the manuscript. F.B., V.D., C.A., C.C., and M.F. were involved in data collection and analysis.

Funding: This research received no external funding.

Acknowledgments: The authors thank Rand Wilcox Vanden Berg for writing assistance and proofreading. DNA samples included in this study originate from NeuroSensCol DNA bank, dedicated for research in neurosensory disorders (PI: JA Sahel, coPI I Audo, partner with CHNO des Quinze-Vingts, Inserm and CNRS).

Conflicts of Interest: The authors declare no conflict of interest.

\section{Abbreviations}

$\begin{array}{ll}\text { MDPI } & \text { Multidisciplinary Digital Publishing Institute } \\ \text { STGD-1 } & \text { Stargardt disease-1 } \\ \text { ABCA4 } & \text { ATP-binding cassette, subfamily A, member 4 } \\ \text { BCVA } & \text { Best Corrected Visual Acuity } \\ \text { ff-ERG } & \text { Full field electroretinography } \\ \text { mf-ERG } & \text { Multi focal electroretinography } \\ \text { SW-AF } & \text { Short-wavelength autofluorescence } \\ \text { NIR-AF } & \text { Near-infrared Autofluorescence } \\ \text { SD-OCT } & \text { Spectral Domain Optical Coherence Tomography } \\ \text { ISCEV } & \text { International Society for Clinical of Electrophysiology of Vision } \\ \text { ACMG } & \text { American College of Medical Genetics and Genomics }\end{array}$




$\begin{array}{ll}\text { AMP } & \text { Association of Molecular Pathology } \\ \text { HGMD } & \text { Human Gene Mutation Database } \\ \text { ExAC } & \text { Exome Aggregation Consortium } \\ \text { PolyPhen2 } & \text { Polymorphism Phenotyping } \\ \text { UCSC } & \text { University of California Santa Cruz } \\ \text { SIFT } & \text { Sorting intolerant from tolerant } \\ \text { RPE } & \text { Retinal pigment epithelium } \\ \text { CRT } & \text { Central retinal thickness } \\ \text { TMV } & \text { Total macular volume } \\ \text { VUS } & \text { Variant of uncertain significance } \\ \text { MAF } & \text { Minor allele frequency } \\ \text { NGS } & \text { Next-generation sequencing } \\ \text { WES } & \text { Whole exome sequencing } \\ \text { WGS } & \text { Whole genome sequencing } \\ \text { EZ } & \text { Ellipsoid zone } \\ \text { FP } & \text { Fundus photograph } \\ \text { FS } & \text { Foveal sparing } \\ \text { MM } & \text { At least one missense variant in the genotype } \\ \text { NM } & \text { At least one null variant in the genotype }\end{array}$

\section{References}

1. Molday, L.L.; Rabin, A.R.; Molday, R.S. ABCR expression in foveal cone photoreceptors and its role in Stargardt macular dystrophy. Nat. Genet. 2000, 25, 257-258. [CrossRef] [PubMed]

2. Maeda, A.; Maeda, T.; Golczak, M.; Palczewski, K. Retinopathy in mice induced by disrupted all-trans-retinal clearance. J. Biol. Chem. 2008, 283, 26684-26693. [CrossRef] [PubMed]

3. Maeda, T.; Maeda, A.; Matosky, M.; Okano, K.; Roos, S.; Tang, J.; Palczewski, K. Evaluation of potential therapies for a mouse model of human age-related macular degeneration caused by delayed all-trans-retinal clearance. Investig. Ophthalmol. Vis. Sci. 2009, 50, 4917-4925. [CrossRef] [PubMed]

4. Koenekoop, R.K. The gene for Stargardt disease, ABCA4, is a major retinal gene: A mini-review. Ophthalmic Genet. 2003, 24, 75-80. [CrossRef] [PubMed]

5. Maia-Lopes, S.; Aguirre-Lamban, J.; Castelo-Branco, M.; Riveiro-Alvarez, R.; Ayuso, C.; Silva, E.D. ABCA4 mutations in Portuguese Stargardt patients: Identification of new mutations and their phenotypic analysis. Mol. Vis. 2009, 15, 584-591. [PubMed]

6. Lewis, R.A.; Shroyer, N.F.; Singh, N.; Allikmets, R.; Hutchinson, A.; Li, Y.; Lupski, J.R.; Leppert, M.; Dean, M. Genotype/Phenotype analysis of a photoreceptor-specific ATP-binding cassette transporter gene, ABCR, in Stargardt disease. Am. J. Hum. Genet. 1999, 64, 422-434. [CrossRef] [PubMed]

7. Martínez-Mir, A.; Paloma, E.; Allikmets, R.; Ayuso, C.; del Rio, T.; Dean, M.; Vilageliu, L.; Gonzàlez-Duarte, R.; Balcells, S. Retinitis pigmentosa caused by a homozygous mutation in the Stargardt disease gene ABCR. Nat. Genet. 1998, 18, 11-12. [CrossRef] [PubMed]

8. Huang, X.; Yuan, L.; Xu, H.; Zheng, W.; Cao, Y.; Yi, J.; Guo, Y.; Yang, Z.; Li, Y.; Deng, H. Identification of a Novel Mutation in the ABCA4 Gene in a Chinese Family with Retinitis Pigmentosa Using Exome Sequencing. Biosci. Rep. 2018. [CrossRef] [PubMed]

9. Audere, M.; Rutka, K.; Šepetiene, S.; Lāce, B. Presentation of Complex Homozygous Allele in ABCA4 Gene in a Patient with Retinitis Pigmentosa. Case Rep. Ophthalmol. Med. 2015, 2015, 452068. [CrossRef] [PubMed]

10. Rozet, J.M.; Gerber, S.; Ghazi, I.; Perrault, I.; Ducroq, D.; Souied, E.; Cabot, A.; Dufier, J.L.; Munnich, A.; Kaplan, J. Mutations of the retinal specific ATP binding transporter gene (ABCR) in a single family segregating both autosomal recessive retinitis pigmentosa RP19 and Stargardt disease: Evidence of clinical heterogeneity at this locus. J. Med. Genet. 1999, 36, 447-451. [PubMed]

11. Mullins, R.F.; Kuehn, M.H.; Radu, R.A.; Enriquez, G.S.; East, J.S.; Schindler, E.I.; Travis, G.H.; Stone, E.M. Autosomal recessive retinitis pigmentosa due to ABCA4 mutations: Clinical, pathologic, and molecular characterization. Investig. Ophthalmol. Vis. Sci. 2012, 53, 1883-1894. [CrossRef] [PubMed] 
12. Cornelis, S.S.; Bax, N.M.; Zernant, J.; Allikmets, R.; Fritsche, L.G.; den Dunnen, J.T.; Ajmal, M.; Hoyng, C.B.; Cremers, F.P.M. In Silico Functional Meta-Analysis of 5,962 ABCA4 Variants in 3,928 Retinal Dystrophy Cases. Hum. Mutat. 2017, 38, 400-408. [CrossRef] [PubMed]

13. Allikmets, R. Simple and complex ABCR: Genetic predisposition to retinal disease. Am. J. Hum. Genet. 2000, 67, 793-799. [CrossRef] [PubMed]

14. Passerini, I.; Sodi, A.; Giambene, B.; Mariottini, A.; Menchini, U.; Torricelli, F. Novel mutations in of the ABCR gene in Italian patients with Stargardt disease. Eye Lond. Engl. 2010, 24, 158-164. [CrossRef] [PubMed]

15. Riveiro-Alvarez, R.; Lopez-Martinez, M.-A.; Zernant, J.; Aguirre-Lamban, J.; Cantalapiedra, D.; Avila-Fernandez, A.; Gimenez, A.; Lopez-Molina, M.-I.; Garcia-Sandoval, B.; Blanco-Kelly, F.; et al. Outcome of ABCA4 disease-associated alleles in autosomal recessive retinal dystrophies: Retrospective analysis in 420 Spanish families. Ophthalmology 2013, 120, 2332-2337. [CrossRef] [PubMed]

16. Jiang, F.; Pan, Z.; Xu, K.; Tian, L.; Xie, Y.; Zhang, X.; Chen, J.; Dong, B.; Li, Y. Screening of ABCA4 Gene in a Chinese Cohort with Stargardt Disease or Cone-Rod Dystrophy with a Report on 85 Novel Mutations. Investig. Ophthalmol. Vis. Sci. 2016, 57, 145-152. [CrossRef] [PubMed]

17. Bocquet, B.; Lacroux, A.; Surget, M.-O.; Baudoin, C.; Marquette, V.; Manes, G.; Hebrard, M.; Sénéchal, A.; Delettre, C.; Roux, A.-F.; et al. Relative frequencies of inherited retinal dystrophies and optic neuropathies in Southern France: Assessment of 21-year data management. Ophthalmic Epidemiol. 2013, 20, 13-25. [CrossRef] [PubMed]

18. Zernant, J.; Schubert, C.; Im, K.M.; Burke, T.; Brown, C.M.; Fishman, G.A.; Tsang, S.H.; Gouras, P.; Dean, M.; Allikmets, R. Analysis of the ABCA4 gene by next-generation sequencing. Investig. Ophthalmol. Vis. Sci. 2011, 52, 8479-8487. [CrossRef] [PubMed]

19. Zernant, J.; Xie, Y.A.; Ayuso, C.; Riveiro-Alvarez, R.; Lopez-Martinez, M.-A.; Simonelli, F.; Testa, F.; Gorin, M.B.; Strom, S.P.; Bertelsen, M.; et al. Analysis of the ABCA4 genomic locus in Stargardt disease. Hum. Mol. Genet. 2014, 23, 6797-6806. [CrossRef] [PubMed]

20. Braun, T.A.; Mullins, R.F.; Wagner, A.H.; Andorf, J.L.; Johnston, R.M.; Bakall, B.B.; Deluca, A.P.; Fishman, G.A.; Lam, B.L.; Weleber, R.G.; et al. Non-exomic and synonymous variants in ABCA4 are an important cause of Stargardt disease. Hum. Mol. Genet. 2013, 22, 5136-5145. [CrossRef] [PubMed]

21. Bauwens, M.; De Zaeytijd, J.; Weisschuh, N.; Kohl, S.; Meire, F.; Dahan, K.; Depasse, F.; De Jaegere, S.; De Ravel, T.; De Rademaeker, M.; et al. An augmented ABCA4 screen targeting noncoding regions reveals a deep intronic founder variant in Belgian Stargardt patients. Hum. Mutat. 2015, 36, 39-42. [CrossRef] [PubMed]

22. Bax, N.M.; Sangermano, R.; Roosing, S.; Thiadens, A.A.H.J.; Hoefsloot, L.H.; van den Born, L.I.; Phan, M.; Klevering, B.J.; Westeneng-van Haaften, C.; Braun, T.A.; et al. Heterozygous deep-intronic variants and deletions in ABCA4 in persons with retinal dystrophies and one exonic ABCA4 variant. Hum. Mutat. 2015, 36, 43-47. [CrossRef] [PubMed]

23. Sangermano, R.; Khan, M.; Cornelis, S.S.; Richelle, V.; Albert, S.; Garanto, A.; Elmelik, D.; Qamar, R.; Lugtenberg, D.; van den Born, L.I.; et al. ABCA4 midigenes reveal the full splice spectrum of all reported noncanonical splice site variants in Stargardt disease. Genome Res. 2018, 28, 100-110. [CrossRef] [PubMed]

24. Maugeri, A.; van Driel, M.A.; van de Pol, D.J.; Klevering, B.J.; van Haren, F.J.; Tijmes, N.; Bergen, A.A.; Rohrschneider, K.; Blankenagel, A.; Pinckers, A.J.; et al. The 2588G $\rightarrow$ C mutation in the ABCR gene is a mild frequent founder mutation in the Western European population and allows the classification of ABCR mutations in patients with Stargardt disease. Am. J. Hum. Genet. 1999, 64, 1024-1035. [CrossRef] [PubMed]

25. Allikmets, R.; Singh, N.; Sun, H.; Shroyer, N.F.; Hutchinson, A.; Chidambaram, A.; Gerrard, B.; Baird, L.; Stauffer, D.; Peiffer, A.; et al. A photoreceptor cell-specific ATP-binding transporter gene (ABCR) is mutated in recessive Stargardt macular dystrophy. Nat. Genet. 1997, 15, 236-246. [CrossRef] [PubMed]

26. Rozet, J.M.; Gerber, S.; Souied, E.; Perrault, I.; Châtelin, S.; Ghazi, I.; Leowski, C.; Dufier, J.L.; Munnich, A.; Kaplan, J. Spectrum of ABCR gene mutations in autosomal recessive macular dystrophies. Eur. J. Hum. Genet. 1998, 6, 291-295. [CrossRef] [PubMed]

27. Schulz, H.L.; Grassmann, F.; Kellner, U.; Spital, G.; Rüther, K.; Jägle, H.; Hufendiek, K.; Rating, P.; Huchzermeyer, C.; Baier, M.J.; et al. Mutation Spectrum of the ABCA4 Gene in 335 Stargardt Disease Patients from a Multicenter German Cohort-Impact of Selected Deep Intronic Variants and Common SNPs. Investig. Ophthalmol. Vis. Sci. 2017, 58, 394-403. [CrossRef] [PubMed] 
28. Salles, M.V.; Motta, F.L.; Dias da Silva, E.; Varela, P.; Costa, K.A.; Filippelli-Silva, R.; Martin, R.P.; Chiang, J.P.-W.; Pesquero, J.B.; Sallum, J.M.F. Novel Complex ABCA4 Alleles in Brazilian Patients with Stargardt Disease: Genotype-Phenotype Correlation. Investig. Ophthalmol. Vis. Sci. 2017, 58, 5723-5730. [CrossRef] [PubMed]

29. Zernant, J.; Lee, W.; Collison, F.T.; Fishman, G.A.; Sergeev, Y.V.; Schuerch, K.; Sparrow, J.R.; Tsang, S.H.; Allikmets, R. Frequent hypomorphic alleles account for a significant fraction of ABCA4 disease and distinguish it from age-related macular degeneration. J. Med. Genet. 2017, 54, 404-412. [CrossRef] [PubMed]

30. Burke, T.R.; Fishman, G.A.; Zernant, J.; Schubert, C.; Tsang, S.H.; Smith, R.T.; Ayyagari, R.; Koenekoop, R.K.; Umfress, A.; Ciccarelli, M.L.; et al. Retinal phenotypes in patients homozygous for the G1961E mutation in the ABCA4 gene. Investig. Ophthalmol. Vis. Sci. 2012, 53, 4458-4467. [CrossRef] [PubMed]

31. Cella, W.; Greenstein, V.C.; Zernant-Rajang, J.; Smith, T.R.; Barile, G.; Allikmets, R.; Tsang, S.H. G1961E mutant allele in the Stargardt disease gene ABCA4 causes bull's eye maculopathy. Exp. Eye Res. 2009, 89, 16-24. [CrossRef] [PubMed]

32. Campa, C.; Gallenga, C.E.; Bolletta, E.; Perri, P. The Role of Gene Therapy in the Treatment of Retinal Diseases: A Review. Curr. Gene Ther. 2017, 17, 194-213. [CrossRef] [PubMed]

33. Lu, L.J.; Liu, J.; Adelman, R.A. Novel therapeutics for Stargardt disease. Graefes Arch. Clin. Exp. Ophthalmol. 2017, 255, 1057-1062. [CrossRef] [PubMed]

34. Webster, A.R.; Héon, E.; Lotery, A.J.; Vandenburgh, K.; Casavant, T.L.; Oh, K.T.; Beck, G.; Fishman, G.A.; Lam, B.L.; Levin, A.; et al. An analysis of allelic variation in the ABCA4 gene. Investig. Ophthalmol. Vis. Sci. 2001, 42, 1179-1189.

35. Allikmets, R.; Shroyer, N.F.; Singh, N.; Seddon, J.M.; Lewis, R.A.; Bernstein, P.S.; Peiffer, A.; Zabriskie, N.A.; Li, Y.; Hutchinson, A.; et al. Mutation of the Stargardt disease gene (ABCR) in age-related macular degeneration. Science 1997, 277, 1805-1807. [CrossRef] [PubMed]

36. Ernest, P.J.G.; Boon, C.J.F.; Klevering, B.J.; Hoefsloot, L.H.; Hoyng, C.B. Outcome of ABCA4 microarray screening in routine clinical practice. Mol. Vis. 2009, 15, 2841-2847. [PubMed]

37. Jimenez-Rolando, B.; Noval, S.; Rosa-Perez, I.; Mata Diaz, E.; Del Pozo, A.; Ibañez, C.; Silla, J.C.; Montaño, V.E.F.; Martin-Arenas, R.; Vallespin, E. Next generation sequencing in the diagnosis of Stargardt's disease. Arch. Soc. Espanola Oftalmol. 2017. [CrossRef]

38. Fujinami, K.; Sergouniotis, P.I.; Davidson, A.E.; Wright, G.; Chana, R.K.; Tsunoda, K.; Tsubota, K.; Egan, C.A.; Robson, A.G.; Moore, A.T.; et al. Clinical and molecular analysis of Stargardt disease with preserved foveal structure and function. Am. J. Ophthalmol. 2013, 156, 487-501.e1. [CrossRef] [PubMed]

39. Souied, E.H.; Ducroq, D.; Rozet, J.M.; Gerber, S.; Perrault, I.; Munnich, A.; Coscas, G.; Soubrane, G.; Kaplan, J. ABCR gene analysis in familial exudative age-related macular degeneration. Investig. Ophthalmol. Vis. Sci. 2000, 41, 244-247.

40. Rivera, A.; White, K.; Stöhr, H.; Steiner, K.; Hemmrich, N.; Grimm, T.; Jurklies, B.; Lorenz, B.; Scholl, H.P.; Apfelstedt-Sylla, E.; Weber, B.H. A comprehensive survey of sequence variation in the ABCA4 (ABCR) gene in Stargardt disease and age-related macular degeneration. Am. J. Hum. Genet. 2000, 67, 800-813. [CrossRef] [PubMed]

41. Sangermano, R.; Bax, N.M.; Bauwens, M.; van den Born, L.I.; De Baere, E.; Garanto, A.; Collin, R.W.J.; Goercharn-Ramlal, A.S.A.; den Engelsman-van Dijk, A.H.A.; Rohrschneider, K.; et al. Photoreceptor Progenitor mRNA Analysis Reveals Exon Skipping Resulting from the ABCA4 c.5461-10T $\rightarrow$ C Mutation in Stargardt Disease. Ophthalmology 2016, 123, 1375-1385. [CrossRef] [PubMed]

42. Birch, D.G.; Peters, A.Y.; Locke, K.L.; Spencer, R.; Megarity, C.F.; Travis, G.H. Visual function in patients with cone-rod dystrophy (CRD) associated with mutations in the ABCA4(ABCR) gene. Exp. Eye Res. 2001, 73, 877-886. [CrossRef] [PubMed]

43. Simonelli, F.; Testa, F.; Zernant, J.; Nesti, A.; Rossi, S.; Allikmets, R.; Rinaldi, E. Genotype-phenotype correlation in Italian families with Stargardt disease. Ophthalmic Res. 2005, 37, 159-167. [CrossRef] [PubMed]

44. Briggs, C.E.; Rucinski, D.; Rosenfeld, P.J.; Hirose, T.; Berson, E.L.; Dryja, T.P. Mutations in ABCR (ABCA4) in patients with Stargardt macular degeneration or cone-rod degeneration. Investig. Ophthalmol. Vis. Sci. 2001, 42, 2229-2236.

45. Klevering, B.J.; Deutman, A.F.; Maugeri, A.; Cremers, F.P.M.; Hoyng, C.B. The spectrum of retinal phenotypes caused by mutations in the ABCA4 gene. Graefes Arch. Clin. Exp. Ophthalmol. 2005, 243, 90-100. [CrossRef] [PubMed] 
46. Fishman, G.A.; Stone, E.M.; Grover, S.; Derlacki, D.J.; Haines, H.L.; Hockey, R.R. Variation of clinical expression in patients with Stargardt dystrophy and sequence variations in the ABCR gene. Arch. Ophthalmol. 1999, 117, 504-510. [CrossRef] [PubMed]

47. Stenirri, S.; Fermo, I.; Battistella, S.; Galbiati, S.; Soriani, N.; Paroni, R.; Manitto, M.P.; Martina, E.; Brancato, R.; Allikmets, R.; et al. Denaturing HPLC profiling of the ABCA4 gene for reliable detection of allelic variations. Clin. Chem. 2004, 50, 1336-1343. [CrossRef] [PubMed]

48. Jaakson, K.; Zernant, J.; Külm, M.; Hutchinson, A.; Tonisson, N.; Glavac, D.; Ravnik-Glavac, M.; Hawlina, M.; Meltzer, M.R.; Caruso, R.C.; et al. Genotyping microarray (gene chip) for the ABCR (ABCA4) gene. Hum. Mutat. 2003, 22, 395-403. [CrossRef] [PubMed]

49. Papaioannou, M.; Ocaka, L.; Bessant, D.; Lois, N.; Bird, A.; Payne, A.; Bhattacharya, S. An analysis of ABCR mutations in British patients with recessive retinal dystrophies. Investig. Ophthalmol. Vis. Sci. 2000, 41, 16-19.

50. Testa, F.; Rossi, S.; Sodi, A.; Passerini, I.; Di Iorio, V.; Della Corte, M.; Banfi, S.; Surace, E.M.; Menchini, U.; Auricchio, A.; et al. Correlation between photoreceptor layer integrity and visual function in patients with Stargardt disease: Implications for gene therapy. Investig. Ophthalmol. Vis. Sci. 2012, 53, 4409-4415. [CrossRef] [PubMed]

51. Aukrust, I.; Jansson, R.W.; Bredrup, C.; Rusaas, H.E.; Berland, S.; Jørgensen, A.; Haug, M.G.; Rødahl, E.; Houge, G.; Knappskog, P.M. The intronic ABCA4 c.5461-10T >C variant, frequently seen in patients with Stargardt disease, causes splice defects and reduced ABCA4 protein level. Acta Ophthalmol. (Copenh.) 2017, 95, 240-246. [CrossRef] [PubMed]

52. Fujinami, K.; Lois, N.; Mukherjee, R.; McBain, V.A.; Tsunoda, K.; Tsubota, K.; Stone, E.M.; Fitzke, F.W.; Bunce, C.; Moore, A.T.; et al. A longitudinal study of Stargardt disease: Quantitative assessment of fundus autofluorescence, progression, and genotype correlations. Investig. Ophthalmol. Vis. Sci. 2013, 54, 8181-8190. [CrossRef] [PubMed]

53. Lois, N.; Holder, G.E.; Bunce, C.; Fitzke, F.W.; Bird, A.C. Phenotypic subtypes of Stargardt macular dystrophy-fundus flavimaculatus. Arch. Ophthalmol. 2001, 119, 359-369. [CrossRef] [PubMed]

54. Fishman, G.A. Fundus flavimaculatus. A clinical classification. Arch. Ophthalmol. 1976, 94, $2061-2067$. [CrossRef] [PubMed]

55. Duncker, T.; Marsiglia, M.; Lee, W.; Zernant, J.; Tsang, S.H.; Allikmets, R.; Greenstein, V.C.; Sparrow, J.R. Correlations among near-infrared and short-wavelength autofluorescence and spectral-domain optical coherence tomography in recessive Stargardt disease. Investig. Ophthalmol. Vis. Sci. 2014, 55, 8134-8143. [CrossRef] [PubMed]

56. Cideciyan, A.V.; Swider, M.; Aleman, T.S.; Sumaroka, A.; Schwartz, S.B.; Roman, M.I.; Milam, A.H.; Bennett, J.; Stone, E.M.; Jacobson, S.G. ABCA4-associated retinal degenerations spare structure and function of the human parapapillary retina. Investig. Ophthalmol. Vis. Sci. 2005, 46, 4739-4746. [CrossRef] [PubMed]

57. Parodi, M.B.; Iacono, P.; Triolo, G.; La Spina, C.; Zucchiatti, I.; Cicinelli, M.V.; Borrelli, E.; Manitto, M.P.; Martina, E.; Bandello, F. Morpho-functional correlation of fundus autofluorescence in Stargardt disease. Br. J. Ophthalmol. 2015, 99, 1354-1359. [CrossRef] [PubMed]

58. Ścieżyńska, A.; Oziębło, D.; Ambroziak, A.M.; Korwin, M.; Szulborski, K.; Krawczyński, M.; Stawiński, P.; Szaflik, J.; Szaflik, J.P.; Płoski, R.; et al. Next-generation sequencing of ABCA4: High frequency of complex alleles and novel mutations in patients with retinal dystrophies from Central Europe. Exp. Eye Res. 2016, 145, 93-99. [CrossRef] [PubMed]

59. Zaneveld, J.; Siddiqui, S.; Li, H.; Wang, X.; Wang, H.; Wang, K.; Li, H.; Ren, H.; Lopez, I.; Dorfman, A.; et al. Comprehensive analysis of patients with Stargardt macular dystrophy reveals new genotype-phenotype correlations and unexpected diagnostic revisions. Genet. Med. 2015, 17, 262-270. [CrossRef] [PubMed]

60. Burke, T.R.; Allikmets, R.; Smith, R.T.; Gouras, P.; Tsang, S.H. Loss of peripapillary sparing in non-group I Stargardt disease. Exp. Eye Res. 2010, 91, 592-600. [CrossRef] [PubMed]

61. Ahn, J.; Beharry, S.; Molday, L.L.; Molday, R.S. Functional interaction between the two halves of the photoreceptor-specific ATP binding cassette protein ABCR (ABCA4). Evidence for a non-exchangeable ADP in the first nucleotide binding domain. J. Biol. Chem. 2003, 278, 39600-39608. [CrossRef] [PubMed]

62. Audo, I.; Manes, G.; Mohand-Saïd, S.; Friedrich, A.; Lancelot, M.-E.; Antonio, A.; Moskova-Doumanova, V.; Poch, O.; Zanlonghi, X.; Hamel, C.P.; et al. Spectrum of rhodopsin mutations in French autosomal dominant rod-cone dystrophy patients. Investig. Ophthalmol. Vis. Sci. 2010, 51, 3687-3700. [CrossRef] [PubMed] 
63. Stenson, P.D.; Ball, E.V.; Mort, M.; Phillips, A.D.; Shiel, J.A.; Thomas, N.S.T.; Abeysinghe, S.; Krawczak, M.; Cooper, D.N. Human Gene Mutation Database (HGMD): 2003 update. Hum. Mutat. 2003, 21, 577-581. [CrossRef] [PubMed]

64. Fokkema, I.F.A.C.; Taschner, P.E.M.; Schaafsma, G.C.P.; Celli, J.; Laros, J.F.J.; den Dunnen, J.T. LOVD v.2.0: The next generation in gene variant databases. Hum. Mutat. 2011, 32, 557-563. [CrossRef] [PubMed]

65. Adzhubei, I.A.; Schmidt, S.; Peshkin, L.; Ramensky, V.E.; Gerasimova, A.; Bork, P.; Kondrashov, A.S.; Sunyaev, S.R. A method and server for predicting damaging missense mutations. Nat. Methods 2010, 7, 248-249. [CrossRef] [PubMed]

66. Sim, N.-L.; Kumar, P.; Hu, J.; Henikoff, S.; Schneider, G.; Ng, P.C. SIFT web server: Predicting effects of amino acid substitutions on proteins. Nucleic Acids Res. 2012, 40, W452-W457. [CrossRef] [PubMed]

67. Schwarz, J.M.; Cooper, D.N.; Schuelke, M.; Seelow, D. MutationTaster2: Mutation prediction for the deep-sequencing age. Nat. Methods 2014, 11, 361-362. [CrossRef] [PubMed]

68. Yeo, G.; Burge, C.B. Maximum entropy modeling of short sequence motifs with applications to RNA splicing signals. J. Comput. Biol. 2004, 11, 377-394. [CrossRef] [PubMed]

69. Reese, M.G.; Eeckman, F.H.; Kulp, D.; Haussler, D. Improved splice site detection in Genie. J. Comput. Biol. 1997, 4, 311-323. [CrossRef] [PubMed]

70. Desmet, F.-O.; Hamroun, D.; Lalande, M.; Collod-Béroud, G.; Claustres, M.; Béroud, C. Human Splicing Finder: An online bioinformatics tool to predict splicing signals. Nucleic Acids Res. 2009, 37, e67. [CrossRef] [PubMed]

71. Casper, J.; Zweig, A.S.; Villarreal, C.; Tyner, C.; Speir, M.L.; Rosenbloom, K.R.; Raney, B.J.; Lee, C.M.; Lee, B.T.; Karolchik, D.; et al. The UCSC Genome Browser database: 2018 update. Nucleic Acids Res. 2017. [CrossRef]

72. Kent, W.J. BLAT-The BLAST-like alignment tool. Genome Res. 2002, 12, 656-664. [CrossRef] [PubMed]

73. Richards, S.; Aziz, N.; Bale, S.; Bick, D.; Das, S.; Gastier-Foster, J.; Grody, W.W.; Hegde, M.; Lyon, E.; Spector, E.; et al. Standards and guidelines for the interpretation of sequence variants: A joint consensus recommendation of the American College of Medical Genetics and Genomics and the Association for Molecular Pathology. Genet. Med. 2015, 17, 405-424. [CrossRef] [PubMed]

74. Marmor, M.F.; Fulton, A.B.; Holder, G.E.; Miyake, Y.; Brigell, M.; Bach, M.; International Society for Clinical Electrophysiology of Vision. ISCEV Standard for full-field clinical electroretinography (2008 update). Doc. Ophthalmol. 2009, 118, 69-77. [CrossRef] [PubMed]

75. Hood, D.C.; Bach, M.; Brigell, M.; Keating, D.; Kondo, M.; Lyons, J.S.; Palmowski-Wolfe, A.M. ISCEV guidelines for clinical multifocal electroretinography (2007 edition). Doc. Ophthalmol. 2008, 116, 1-11. [CrossRef] [PubMed]

76. Schmitz-Valckenberg, S.; Brinkmann, C.K.; Alten, F.; Herrmann, P.; Stratmann, N.K.; Göbel, A.P.; Fleckenstein, M.; Diller, M.; Jaffe, G.J.; Holz, F.G. Semiautomated image processing method for identification and quantification of geographic atrophy in age-related macular degeneration. Investig. Ophthalmol. Vis. Sci. 2011, 52, 7640-7646. [CrossRef] [PubMed]

77. Strauss, R.W.; Muñoz, B.; Wolfson, Y.; Sophie, R.; Fletcher, E.; Bittencourt, M.G.; Scholl, H.P.N. Assessment of estimated retinal atrophy progression in Stargardt macular dystrophy using spectral-domain optical coherence tomography. Br. J. Ophthalmol. 2016, 100, 956-962. [CrossRef] [PubMed]

78. Parker, M.A.; Choi, D.; Erker, L.R.; Pennesi, M.E.; Yang, P.; Chegarnov, E.N.; Steinkamp, P.N.; Schlechter, C.L.; Dhaenens, C.-M.; Mohand-Said, S.; et al. Test-Retest Variability of Functional and Structural Parameters in Patients with Stargardt Disease Participating in the SAR422459 Gene Therapy Trial. Transl. Vis. Sci. Technol. 2016, 5, 10. [CrossRef] [PubMed]

79. Lee, W.; Xie, Y.; Zernant, J.; Yuan, B.; Bearelly, S.; Tsang, S.H.; Lupski, J.R.; Allikmets, R. Complex inheritance of ABCA4 disease: Four mutations in a family with multiple macular phenotypes. Hum. Genet. 2016, 135, 9-19. [CrossRef] [PubMed]

(C) 2018 by the authors. Licensee MDPI, Basel, Switzerland. This article is an open access article distributed under the terms and conditions of the Creative Commons Attribution (CC BY) license (http:/ / creativecommons.org/licenses/by/4.0/). 